Z Gerontol Geriat 2011 [Suppl 2] · 44:9-25

DOI 10.1007/s00391-011-0248-4

Online publiziert: 2. Dezember 2011

(c) Springer-Verlag 2011

\author{
B. Schüz ${ }^{1}$ D. Dräger ${ }^{2} \cdot$ S. Richter ${ }^{3} \cdot$ K. Kummer ${ }^{2}$ A. Kuhlmey² - C. Tesch-Römer ${ }^{4}$ \\ ${ }^{1}$ School of Psychology, University of Tasmania, Tasmania \\ ${ }^{2}$ Institut für Medizinische Soziologie, Charité - Universitätsmedizin Berlin \\ ${ }^{3}$ 2008-2010 AMA-Verbund, Charité, Fachhochschule Jena \\ ${ }^{4}$ Deutsches Zentrum für Altersfragen, Berlin
}

\title{
Autonomie trotz Multimorbidität im Alter - Der Berliner Forschungsverbund AMA
}

gehen die Forscher ${ }^{1}$ (• Tab. 1) des Berliner Verbundes „Autonomie trotz Multimorbidität im Alter“ (AMA) in insgesamt sechs Forschungsprojekten nach.

\section{Multimorbidität, Autonomie und Lebensqualität}

Für Deutschland zeigen repräsentative Daten des Deutschen Alterssurveys (DEAS; [95]), dass der Anteil der Personen, die multimorbid erkrankt sind (also zwei oder mehr Erkrankungen aufweisen), von $62 \%$ in der Gruppe der 64- bis 69-Jährigen auf $74 \%$ in der Gruppe der 70- bis 75-Jährigen und 80\% in der Gruppe der 76- bis 81-Jährigen ansteigt. Das bedeutet, dass der Großteil der älteren Menschen in Deutschland unter multiplen Erkrankungen leidet. Außerdem gilt für Gesundheit im Alter, wie für fast alle anderen Lebensbereiche auch, dass sich in dieser Lebensphase große Unterschiede zwischen Menschen zeigen [94], was allgemeingültige Aussagen über den Gesundheitszustand im Alter und Ressourcen für Gesundheit im Alter zu einer großen Herausforderung macht.

Bisher fehlt eine einheitliche Definition der Multimorbidität, die über das gleichzeitige Vorhandensein von mehreren Erkrankungen hinausgeht, obwohl viele Arbeiten betonen, dass sich Multi-

1 Aus Gründen der besseren Lesbarkeit wird im Text zumeist die maskuline Form der Substantive verwendet. In der Regel sind damit beide Geschlechter gemeint. morbidität massiv auf alle Bereiche des täglichen Lebens auswirkt $[31,33,34,74$, 77, 90].

Das Vorliegen von mehreren Erkrankungen erhöht das Risiko, unter Gebrechlichkeit oder frailty zu leiden, was sich auf die funktionale Gesundheit und so auf die Möglichkeiten zur selbständigen Gestaltung des alltäglichen Lebens auswirkt [33]. Diese Einschränkungen in der Gestaltung des täglichen Lebens, verbunden mit krankheitsspezifischen Beschwerden wie Schmerzen, führen dazu, dass multimorbide Menschen eine niedrigere Lebensqualität berichten [30, 31], und zwar sowohl in Bezug auf die Gesundheit als auch auf die allgemeine Lebensqualität.

Neben diesen Einschränkungen der Lebensqualität können Multimorbidität und Gebrechlichkeit auch die Autonomie älterer Menschen einschränken. Autonomie geht dabei über den bloßen Begriff von Selbständigkeit oder Selbstversorgung hinaus. Viele ältere Menschen mit Mehrfacherkrankungen sind zumindest in Teilen ihrer Aktivitäten des täglichen Lebens durch funktionale Behinderungen eingeschränkt [84]. Daher wird Autonomie im gerontologischen Kontext weiter gefasst und setzt das Vorhandensein von Wahlmöglichkeiten voraus [76] - ältere Menschen mit Mehrfacherkrankungen sind vor allem dann autonom, wenn sie selbst wählen und über Din-

Der AMA-Verbund wird vom Bundesministerium für Bildung und Forschung gefördert (BMBF, Förderkennzeichen 01ET0701 bis 01ET0707) ein Leben in hoher Lebensqualität und Autonomie führen können. Dieser Frage 
Tab. 1 Mitarbeiterinnen und Mitarbeiter der sieben Teilprojekte des Berliner Forschungsverbundes „Autonomie trotz Multimorbidität im Alter"

\begin{tabular}{|c|c|}
\hline Projekt & Mitarbeiterinnen und Mitarbeiter \\
\hline AMA-INTEGRATION & $\begin{array}{l}\text { Uwe Flick, Adelheid Kuhlmey, Peter Martus, Stefanie Richter, Matthias Riepe, } \\
\text { Gundula Röhnsch, Sandra Schrei, Andrea Stroux, Clemens Tesch-Römer }\end{array}$ \\
\hline OMAHA & $\begin{array}{l}\text { Markus Busch, Andrea Ernert, Judith Fuchs, Beate Gaertner, Ulfert Hapke, } \\
\text { Martin Holzhausen, Hiltraud Knopf, Peter Martus, Christa Scheidt-Nave, } \\
\text { Justus Welke }\end{array}$ \\
\hline PREFER & $\begin{array}{l}\text { Benjamin Schüz, Ralf Schwarzer, Lisa M. Warner, Clemens Tesch-Römer, } \\
\text { Susanne Wurm, Jochen P. Ziegelmann }\end{array}$ \\
\hline MIGRANT-DEM & $\begin{array}{l}\text { Ertan Altintas, Ulrike Beinhoff, Rana Kalkan, Matthias Riepe, } \\
\text { Zaruhi Stepanyan }\end{array}$ \\
\hline NEIGHBOURHOOD & $\begin{array}{l}\text { Katrin Falk, Anna Herzog, Josefine Heusinger, Kerstin Kammerer, } \\
\text { Meggi Khan-Zvorničanin, Susanne Kümpers, Kathrin Ottovay, } \\
\text { Clara Wenger-Haargassner, Maria Wöpking, Michael Zander }\end{array}$ \\
\hline INSOMNIA & $\begin{array}{l}\text { Uwe Flick, Vjenka Garms-Homolová, Joachim Kuck, Silke Migala, } \\
\text { Gundula Röhnsch, Katrin Theiss }\end{array}$ \\
\hline PAIN & $\begin{array}{l}\text { Dagmar Dräger, Michaela Golz, Yvonne Hallo, Sebastian Ellert, } \\
\text { Thomas Fischer, Sonja Kalinowski, Marita Kölzsch, Kirsten Kopke, } \\
\text { Ronny Kuhnert, Reinhold Kreutz, Ines Wulff }\end{array}$ \\
\hline
\end{tabular}

ge des täglichen Lebens bestimmen können. Autonomie bedeutet also nicht unbedingt, alles selbständig erledigen zu können. Autonom handeln kann beispielsweise auch umfassen, sich bewusst für einen bestimmten Pflegedienst zu entscheiden oder auch zwischen verschiedenen Mahlzeiten im Pflegeheim auszuwählen. Diese Konzeption von Autonomie macht deutlich, dass sie sich nicht nur in funktionalen Indikatoren, sondern auch in subjektiven Einschätzungen von Handlungsspielräumen niederschlägt $[47,83]$. Das Erleben von Wahlmöglichkeiten ist allerdings eng an die gesundheitlichen Voraussetzungen älterer Menschen geknüpft und wird durch Multimorbidität bedroht [84].

Die Herausforderungen, die Multimorbidität an Lebensqualität und Autonomie stellt, machen auch deutlich, dass große Unterschiede zwischen verschiedenen Gruppen älterer Menschen und zwischen Individuen zu erwarten sind. Tatsächlich zeigt sich, dass sich Krankheiten und Einschränkungen nicht gleichförmig auf Lebensqualität und Autonomie älterer Menschen auswirken, sondern dass viele verschiedene Ressourcen dafür verantwortlich sein können, wie ältere Menschen Multimorbidität in Einklang mit dem Streben nach Lebensqualität und Autonomie bringen [5, 29, 94].

\section{Ressourcen für Lebensqualität und Autonomie trotz Multimorbidität}

Die Lebensformen alter Menschen sind sehr different, dies trifft auch auf die Lebensformen alter Frauen und Männer mit Mehrfacherkrankungen zu [94]. Daraus resultiert, dass mehrfach erkrankte Menschen Faktoren, die für sie Ressourcen für Autonomie und Lebensqualität sein könnten, sehr unterschiedlich bewerten.

Ressourcen können auf verschiedenen Ebenen verortet sein und von unterschiedlichen Quellen ausgehen. Außerdem lassen sich Ressourcen erst durch einen definierten Endzustand begreifen - eine Ressource ist erst dann eine Ressource, wenn Menschen durch sie einen bestimmten Zustand erreichen können [13]. Zum Beispiel wird allgemein davon ausgegangen, dass soziale Unterstützung vor allem bei Menschen mit gesundheitlichen Einschränkungen eine wichtige Ressource für Lebensqualität und Gesundheit darstellt [89]. Gleichzeitig zeigen Studien, dass soziale Unterstützung auch als Einschränkung der Autonomie und Entscheidungsfreiheit erlebt werden kann [18]. Soziale Unterstützung kann also abhängig von individuellen Voraussetzungen entweder eine Ressource oder ein Risikofaktor für Lebensqualität bzw. Autonomie sein.

Das macht deutlich, dass in der Forschung zu Ressourcen für Lebensqua- lität und Autonomie bei Multimorbidität nicht davon auszugehen ist, dass bestimmte Faktoren in jedem Fall Ressourcen darstellen, sondern dass die individuellen, sozialen, sozialräumlichen und institutionellen Rahmungen, in denen Menschen leben, dafür verantwortlich sind, ob bestimmte Faktoren tatsächlich Ressourcen darstellen.

Ressourcen lassen sich also auf mehreren Ebenen, von der individuellen bis hin zur Versorgungslandschaft, lokalisieren. Zum Beispiel können individuelle Ressourcen, wie die Bildung eines Menschen [4] oder seine Selbstwirksamkeit [6], das Verhalten eines Multimorbiden so beeinflussen, dass sie unterstützend auf die Autonomie trotz Erkrankungen wirken. Konkret kann beispielsweise Bildung dabei helfen, bessere Entscheidungen über ambulante Versorgungsmöglichkeiten $\mathrm{zu}$ treffen [71], und Selbstwirksamkeit kann ältere Menschen dabei unterstützen, länger körperlich aktiv zu bleiben $[5,92]$. Zudem entscheiden finanzielle Ressourcen darüber, ob kostenpflichtige Angebote und Hilfen in Anspruch genommen werden [78].

Des Weiteren können ungleich verteilte Ressourcen in der direkten Umgebung älterer Menschen zu gesundheitlicher Ungleichheit beitragen [10]. Unterschiede im Angebot von Waren des täglichen Bedarfs und gesundheitlicher Versorgung zwischen dicht besiedelten städtischen und spärlich bewohnten ländlichen Regionen können beispielsweise für ältere Menschen mit Einschränkungen in der Mobilität entscheidend dafür sein, wie gut oder wie schlecht sie sich selbst versorgen können.

Für ältere Menschen mit Mehrfacherkrankungen, die in Pflegeheimen leben, sind neben personengebundenen Faktoren die Gegebenheiten in der Einrichtung wichtige Ressourcen. Das umfasst neben der baulichen Gestaltung und der Personalausstattung der Heime auch Faktoren wie die Ausbildung des Personals oder die Orientierung an Richtlinien zur Medikamentengabe [55]. 


\section{Der Berliner Forschungsverbund AMA}

Vor diesem Hintergrund stellte sich der Berliner Forschungsverbund AMA die Leitfrage, inwieweit eine selbstbestimmte Lebensgestaltung alter Menschen trotz Mehrfacherkrankung und funktioneller Beeinträchtigungen erhalten und gefördert werden kann. Dementsprechend verfolgte der interdisziplinäre und praxisbezogene AMA-Verbund drei Ziele:

1. Die Bereitstellung wissenschaftlicher Befunde zur Verbesserung der Versorgung und Alltagsbewältigung alter und hochaltriger Menschen mit Mehrfacherkrankungen sowie zur Prävention von Mehrfacherkrankungen bzw. der damit einhergehenden Gesundheitsprobleme Schmerz, Schlafstörungen und Stürze.

2. Die Erarbeitung eines konzeptionellen Rahmens zur mehrdimensionalen Erfassung von Multimorbidität und Autonomie sowie die Bereitstellung entsprechender standardisierter Instrumente für bevölkerungsbezogene Studien.

3. Den Aufbau nachhaltiger gerontologischer Forschungs- und Qualifizierungsstrukturen in Berlin.

Mehr zu den Projekten unter http://www. ama-consortium.de

Insgesamt waren am Forschungsverbund AMA zehn Berliner Wissenschaftseinrichtungen beteiligt. In fast allen Teilprojekten ( $\bullet$ Tab. 2) kooperierten wiederum mindestens zwei Einrichtungen bei der Bearbeitung der Projektfragestellung.

Im gesamten Förderzeitraum arbeiteten in den sieben Projekten des AMAVerbundes etwa 50 Wissenschaftler unterschiedlicher Disziplinen zusammen. Darüber hinaus wurde über die Einbeziehung von sieben Doktoranden eine enge Verbindung zwischen dem AMA-Verbund und dem Graduiertenkolleg der Charité „Multimorbidität im Alter“ (gefördert von der Robert Bosch Stiftung) erreicht.

Die interdisziplinäre Zusammensetzung der Forschergruppen erforderte eine kontinuierliche Kommunikation, die eine Verständigung über Diskurse einzelner Disziplinen ermöglichte und zur Per-

\section{Z Gerontol Geriat 2011 · 44:9-25 ～DOI 10.1007/s00391-011-0248-4 \\ (c) Springer-Verlag 2011}

\section{B. Schüz · D. Dräger · S. Richter · K. Kummer · A. Kuhlmey · C. Tesch-Römer Autonomie trotz Multimorbidität im Alter - Der Berliner Forschungsverbund AMA}

\section{Zusammenfassung}

Mit zunehmendem Alter steigt der Anteil mehrfach erkrankter Menschen und immer mehr Menschen erreichen ein sehr hohes Lebensalter. Trotz des medizinischen Fortschritts und verbesserter Lebenslagen müssen viele alte Menschen körperliche, psychische und soziale Einschränkungen bewältigen. Es ist eine große gesundheits- und sozialpolitische Herausforderung, diese alten Frauen und Männer mit gesundheitlichen Beeinträchtigungen in ihrem Bestreben nach einer weitestgehend autonomen Lebensgestaltung zu unterstützen und zu stärken.

Vor diesem Hintergrund widmete sich der Forschungsverbund "Autonomie trotz Multimorbidität im Alter" (AMA I) der Leitfrage, inwieweit eine selbstbestimmte Lebensgestaltung alter mehrfach erkrankter Menschen erhalten werden kann. Um der Vielfalt der Lebenswelten älterer Menschen gerecht zu werden, wurden im Alltag wenig eingeschränkte und pflegebedürftige sowie ko- gnitiv beeinträchtigte Personen in die Untersuchung einbezogen. Darunter waren Frauen und Männer, die noch im eigenen Haushalt und solche die im Pflegeheim lebten. Im Fokus der Untersuchungen standen die Ressourcen, die den alten Menschen in ihrer jeweiligen Lebenssituation zur Verfügung standen und die Frage, wie diese gestärkt werden können. Der Beitrag präsentiert Ergebnisse zu dieser Forschungsfrage aus der 1. Förderphase des AMA-Verbundes. Darauf aufbauend werden in einer 2. Förderphase (20112013, AMA II) nunmehr nachhaltige praxisrelevante Interventionen zur Mobilisierung von autonomierelevanten Ressourcen mehrfach erkrankter alter Menschen entwickelt und auf ihre Praxistauglichkeit getestet.

\section{Schlüsselwörter}

Alter - Autonomie $\cdot$ Multimorbidität .

Lebensqualität · Ressourcen · Schmerz ·

Schlafstörungen · Sturz

\section{Autonomy despite multimorbidity in old age - The Berlin-based AMA research consortium}

\section{Abstract}

The proportion of the population with multiple illnesses increases with age and growing numbers of people are now living to a very old age. Despite medical progress and improved living conditions, many old people have to deal with physical, psychological, and social impairments. It is a crucial challenge for health and social policy to support the elderly with health-related impairments in their desire to lead as independent a life as possible.

Against this background the research consortium Autonomy Despite Multimorbidity in Old Age (AMA I) examined the extent to which the self-determined life style of multimorbid old and very old persons can be supported and maintained. In order to reflect the diversity of life worlds of the elderly, the study sample included participants who were not notably impaired in their everyday functioning, participants in need of nursing care and participants with cognitive impairments.
Moreover, the sample comprised both older persons who were still living in their own homes and nursing home residents. The studies conducted within the AMA framework focused on the resources available to old persons living in different situations and on how these resources can be strengthened. This article presents findings from the first phase of funding of the AMA research consortium. In a second phase of funding (2011-2013, AMA II), sustainable practice-based interventions are being developed to mobilize resources which can help multimorbid older persons to maintain their autonomy and the practical viability of these interventions will be tested.

\section{Keywords}

Old age $\cdot$ Autonomy $\cdot$ Multimorbidity .

Quality of life - Resources · Pain .

Sleep disorders $\cdot$ Risk of falling 
Tab. 2 Übersicht der Verbundprojekte des Berliner Forschungsverbundes AMA

Projekt
OMAHA*:
Operationalisierung von Multi-
morbidität und Autonomie für
die Versorgungsforschung in
alternden Populationen

Partner

- Robert Koch-Institut (RKI)

- Charité - Universitätsmedizin Berlin

\section{MIGRANT-DEM:}

Demenz und Multimorbidität bei Migranten mit nicht deutscher Muttersprache im urbanen Raum

\section{PREFER:}

Personale Ressourcen von älteren Menschen mit Mehrfacherkrankungen: Stärkung effektiven Gesundheitsverhaltens

\section{NEIGHBOURHOOD:}

Autonomieerhalt angesichts von Sturzfolgen in sozial benachteiligten Quartieren und Nachbarschaften

INSOMNIA:

Schlafstörungen und Multimorbidität in der stationären Langzeitpflege von alten Menschen

\section{PAIN:}

Autonomie trotz Schmerz - Schmerzgeschehen und Schmerzmedikation bei Pflegeheimbewohnern

\section{AMA-INTEGRATION:} Koordination, Methodenberatung und wissenschaftliche Integration

\author{
- Alice Salomon Hochschule Berlin \\ (ASH)
}

- Charité - Universitätsmedizin Berlin

- Charité - Universitätsmedizin Berlin

\author{
- Charité - Universitätsmedizin Berlin \\ - Universität UIm
}

- Deutsches Zentrum für Altersfragen (DZA)

- Freie Universität Berlin

\section{Inhalte}

- Epidemiologie von Mehrfacherkrankungen (Multimorbidität)

- Konzeptionelle Weiterentwicklung des Konstrukts Multimorbidität

- Entwicklung eines Sets an Kernindikatoren, Erhebungsinstrumenten und Auswertungsstrategien zur Erfassung von Multimorbidität, Autonomie, Lebensqualität

- Bestimmung von Determinanten von Gesundheit, von Präventionspotenzialen und des spezifischen Versorgungsbedarfs

- Bevölkerungsrepräsentative Längsschnittstudie; standardisiertes Design

- Population: Menschen ab 65 Jahren in Privathaushalten

- Demenzielle Erkrankungen und Mehrfacherkrankungen

- Versorgungsstrukturen und Inanspruchnahmeverhalten von Menschen mit Migrationshintergrund (Identifizierung von Barrieren)

- Entwicklung sprachunabhängiger diagnostischer Instrumente

- Standardisiertes Design

- Population: Menschen ab 65 Jahren mit türkischem und russischem Migrationshintergrund und kognitiven Problemen; Beratungssetting

- Personale Ressourcen (z. B. Kompetenzüberzeugungen, Vorstellung über das Älterwerden, selbstbestärkende Kognitionen, Gesundheitsverhalten) und soziale Ressourcen (soziale Unterstützung)

- Längsschnittstudie, verknüpft mit dem Deutschen Alterssurvey; standardisiertes Design

- Population: Menschen ab 65 Jahren mit Mehrfacherkrankungen in Privathaushalten

- Sozialräumliche Ressourcen (z. B. professionelle Infrastruktur, Barrierefreiheit, Mobilitätsangebote) und individuelle Ressourcen (Milieu, Bildung, materielle Ressourcen und Inanspruchnahme von Leistungen)

- Interpretatives Design (Interviews, Stadtteilbeobachtung, Fokusgruppen)

- Population: mehrfach erkrankte alte Menschen mit Sturzfolgen in sozial benachteiligten Quartieren

- Epidemiologie von Schlafstörungen bei Mehrfacherkrankungen

- Institutionelle Ressourcen (Lebensraumgestaltung wie Licht, Tagesregime, Aktivitätsangebote) und professionelle Ressourcen (Problembewusstsein, Fachwissen, Strategien und Schlafförderung)

- Längsschnittstudie mit standardisiertem Design (Interviews, Beobachtungen)

- Population: pflegebedürftige Menschen und Professionelle in Einrichtungen der stationären Langzeitpflege

- Epidemiologie von Schmerzen bei Mehrfacherkrankungen

- Professionelle Ressourcen (Qualität, und Angemessenheit der Schmerzmedikation sowie pflegerischer und therapeutischer Interventionen)

- Querschnittstudie (Primär-, Sekundärdaten); standardisiertes Design

- Population: pflegebedürftige Menschen und Professionelle in Einrichtungen der stationären Langzeitpflege - Deutsches Zentrum für Altersfragen - wissenschaftliche Koordination, Wissensintegration, Methodenberatung Öffentlichkeitsarbeit, Dissemination
(DZA)

- Alice Salomon Hochschule Berlin

(ASH)

*Kürzel ergeben sich aus den englischen Projekttiteln

spektiverweiterung sowie zum Wissenstransfer beitrug. Diese Aufgabe moderierte das siebte Teilprojekt AMA-INTEGRA$\mathrm{TION}^{2}$ über Strukturelemente, die den projekt- und disziplinenübergreifenden Austausch förderten und der Wissensin-

2 AMA-INTEGRATION: BMBF Förderkennzeichen - 01 ET0701 tegration diente. Dazu gehörten projektübergreifende themenbezogene Arbeitsgruppen, Methodenworkshops, inhaltliche Workshops, Arbeitsbesprechungen sowie Projektbegehungen [73]. Die Diskussion der Herausforderungen disziplinübergreifender Zusammenarbeit im Verbund und möglicher Strategien zur Be- wältigung von Hindernissen findet sich in Richter et al. [74].

In den vergangenen Jahren erfolgte in der gerontologischen Forschung eine Erweiterung der Perspektive von einem defizitorientierten Fokus hin zur Identifizierung und Untersuchung von Ressourcen, die ein gutes Leben im Alter ermöglichen [5]. Daher nimmt der Berliner For- 
schungsverbund AMA dezidiert eine ressourcenorientierte Perspektive ein und untersucht die Ressourcen, die es älteren Menschen mit Multimorbidität ermöglichen, mit Lebensqualität und Autonomie zu leben. Um der Vielfalt der Lebenswelten älterer Menschen mit Mehrfacherkrankungen in Deutschland gerecht zu werden, untersuchte der Verbund Ressourcen bei Menschen, die noch relativ selbständig im eigenen Haushalt leben bis hin zu schwer pflegebedürftigen und kognitiv beeinträchtigen Menschen im Pflegeheim (• Abb. 1).

\section{Methoden und Ergebnisse}

Im Folgenden werden die Methoden und ausgewählte Ergebnisse der Teilprojekte des AMA-Verbundes am Beispiel einer konkreten Forschungsfrage vorgestellt. Weitere Ergebnisse aus den Projekten finden sich in den erwähnten weiterführenden Publikationen.

\section{Personale Ressourcen für Lebensqualität und Autonomie (Projekt PREFER) $^{3}$}

\section{Benjamin Schüz, Ralf Schwarzer, Clemens Tesch-Römer, Lisa M. Warner, Susanne Wurm, Jochen P. Ziegelmann ${ }^{4}$}

Das Projekt PREFER (Personale Ressourcen für Autonomie und Lebensqualität bei älteren Menschen mit Mehrfacherkrankungen) deckt im Rahmen der Projekte des AMA-Verbundes die Population älterer Menschen mit Mehrfacherkrankungen ab, die noch im eigenen Haushalt leben ( Abb. 1). PREFER ging der Frage nach, welche personengebundenen Ressourcen dafür verantwortlich sind, dass ältere Menschen mit Mehrfacherkrankungen ein selbstbestimmtes Leben in Autonomie und hoher Lebensqualität verbringen können.

\section{Methoden}

Im Rahmen der PREFER-Studie sollten vor allem Wechselwirkungen zwischen

\footnotetext{
3 PREFER: BMBF Förderkennzeichen 01ET0702

4 Die Autoren aller Projektkapitel sind in alphabetischer Reihenfolge angeführt.
}

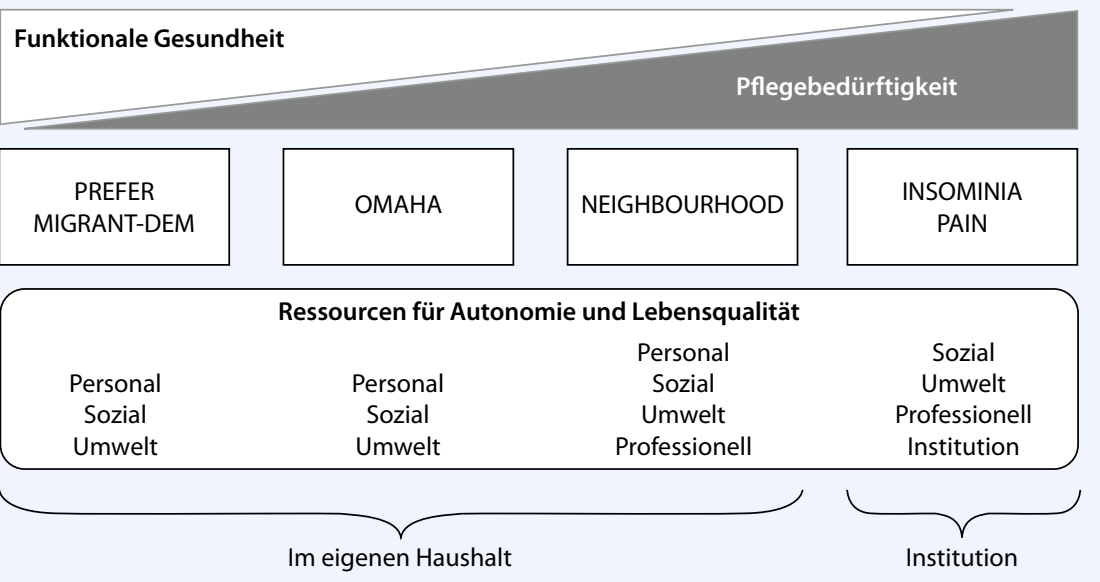

Abb. $1 \Delta$ Projekte des AMA-Verbundes auf einem hypothetischen Kontinuum von funktionaler Gesundheit und Pflegebedürftigkeit

personalen Ressourcen, gesundheitlich relevanten Verhaltensweisen, Gesundheit und Autonomie untersucht werden. PREFER wurde daher als längsschnittliche Studie mit drei Messzeitpunkten über einen Zeitraum von sechs Monaten konzipiert. Die Stichprobe für die PREFER-Studie wurde über Gesundheitsmessungen aus den Teilnehmern des Deutschen Alterssurveys (DEAS; [95]) rekrutiert. Einschlusskriterien waren neben dem Vorliegen von mindestens zwei Erkrankungen aus dem Charlson-Comorbidity-Index [17] oder dem Functional-Comorbidity-Index [44] die Einwilligung, für weitere Studien kontaktiert zu werden und ein Alter von mindestens 65 Jahren. Daraus ergab sich aus der Gesamtstichprobe des DEAS eine Substichprobe von $\mathrm{n}=309$ Personen, die über den Untersuchungszeitraum dreimal befragt wurden. Zudem lagen für alle Personen weitere Informationen zu einem vierten Messzeitpunkt vor, der im Rahmen des Deutschen Alterssurveys stattfand.

Die Teilnehmer waren durchschnittlich 73,27 Jahre alt $(S D=5,1)$. Etwas mehr als die Hälfte der Teilnehmer waren Männer $(58,3 \%)$. Die Teilnehmenden leben über das ganze Gebiet der Bundesrepublik verteilt $-65,05 \%$ gaben an, in den alten und $34,95 \%$ gaben an, in den neuen Bundesländern zu leben. Im Schnitt litten die Teilnehmenden unter 5,55 Erkrankungen (Erfassung im Selbstbericht), wobei vor allem erhöhter Blutdruck und Arthrose bei mehr als der Hälfte der teilnehmenden Personen vorkamen. Zum ersten Messzeitpunkt nahmen 309 Personen an der PREFER-Studie teil, zum zweiten Messzeitpunkt (schriftliche Befragung) konnten 257 Personen $(83,17 \%)$ und zum dritten Messzeitpunkt (persönliche und schriftliche Befragung) 278 Personen (89,97\% der Ausgangsstichprobe) erreicht werden. Die Teilnahme oder Nicht-Teilnahme am zweiten und dritten Messzeitpunkt konnte durch keine der in der Studie erhobenen Variablen in logistischen Regressionsanalysen vorhergesagt werden (alle $p s>0,05$ ), daher kann angenommen werden, dass die längsschnittliche Stichprobe repräsentativ für die gesamte PREFER-Stichprobe ist.

\section{Ergebnisse}

Die Analyse der Daten der PREFER-Studie hat auf mehreren Ebenen und in verschiedenen Bereichen Ergebnisse erbracht, die für die Verbesserung von Gesundheit, Lebensqualität und Autonomie älterer Menschen mit Mehrfacherkrankungen bedeutsam sein können.

In der PREFER-Studie wurde unter anderem untersucht, welche persönlichen Ressourcen für körperliche Aktivität [93], Medikamentenadhärenz [80, 82], aber auch für das Erleben von Autonomie [92] wichtig sind. Auf letzteren Befund soll im Folgenden etwas näher eingegangen werden. Eine umfassendere Diskussion der Ergebnisse findet sich in Schüz et al. [81].

Wie oben angeführt, umfasst der Begriff der Autonomie im Alter sowohl Selbständigkeit als auch Selbstbestimmung. Im Rahmen der PREFER-Studie war vor allem das subjektive Erleben von Autonomie von Interesse, das sich in relati- 


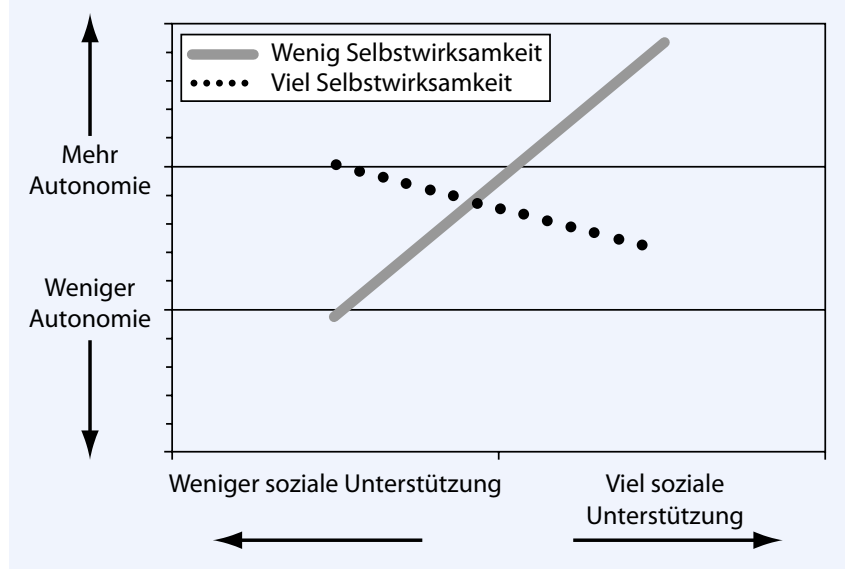

Abb. $2<$ Schematische Darstellung der Interaktion von Selbstwirksamkeit und sozialer Unterstützung in der Vorhersage von Autonomie ver Unabhängigkeit von objektiven Indikatoren der Selbständigkeit wie Aktivitäten des täglichen Lebens [57] entwickeln kann. Dazu wurde eine neue Skala zur Erfassung von Autonomieerleben entwickelt (WAA; [83]), in der mit vier Items die Dimensionen Selbständigkeit, Selbstbestimmung und gedankliche Autonomie erfasst werden. Diese Dimensionen finden sich zwar auch in anderen Instrumenten zur Erfassung von Autonomie wieder (z. B. $[29,47])$, sind aber meist recht umfänglich und sprachlich wenig ökonomisch erfasst.

Frühere Studien haben gezeigt, dass sich ältere Menschen vor allem dann autonom im Sinne von hoher Selbstbestimmung fühlen, wenn sie davon überzeugt sind, dass sie den Lauf ihres weiteren Lebens aus eigener Kraft meistern und kontrollieren können (Selbstwirksamkeit; [6]). Zudem ist vor allem im Angesicht von zunehmenden funktionalen Einschränkungen Unterstützung aus dem sozialen Umfeld eine wichtige Ressource für das Leben im Alltag [89] und kann so dazu beitragen, dass ältere Menschen auch mit Einschränkungen ein selbstbestimmtes Leben führen können. Diese beiden Ressourcen stehen in einem Spannungsverhältnis, weil soziale Unterstützung schnell als Überbehütung erlebt wird [18] oder zu niedrigerem Selbstwert auf der Seite des Empfängers führen kann [89]. Daher ist die Frage, in welchem Verhältnis Selbstwirksamkeit und soziale Unterstützung in der Erklärung von Autonomie stehen, wichtig.

Um diese Frage zu beantworten, wurde wahrgenommene Autonomie durch Selbstwirksamkeit und soziale Unterstützung sowie die Interaktion dieser beiden Variablen im Längsschnitt erklärt. Es zeig- te sich, dass der Interaktionsterm aus sozialer Unterstützung und Selbstwirksamkeit einen signifikanten Beitrag zur Erklärung von erlebter Autonomie leisten kann. Die Detaildarstellung der Interaktion zeigte, dass Menschen mit niedriger Selbstwirksamkeit die negativen Auswirkungen von niedriger Selbstwirksamkeit auf Autonomie durch soziale Unterstützung kompensieren können. Auf der anderen Seite erlebten Menschen mit hoher Selbstwirksamkeit viel soziale Unterstützung als Einschränkung ihrer Autonomie (- Abb. 2).

Diese Befunde legen nahe, dass Ressourcen auf der Ebene der Person miteinander interagieren und wichtige Voraussetzungen für Verbesserungen der Autonomie im Alter sein können, wenn sie gezielt und in Abhängigkeit von den individuellen Voraussetzungen und Bedürfnissen der Person gefördert werden [92].

\section{Erreichbarkeit und Lebensqualität in alternden, multimorbid erkrankten Populationen (Projekt OMAHA) $^{5}$}

\section{Martin Holzhausen}

Das Projekt OMAHA (Operationalisierung von Multimorbidität und Autonomie für die Versorgungsforschung in alternden Populationen) deckt im Rahmen des AMA-Verbundes die Zielgruppe älterer Personen im eigenen Haushalt ab, die bereits unter beginnender Gebrechlichkeit [33] und funktionalen Einschränkungen leiden (• Abb. 1). OMAHA ging ins-

\footnotetext{
5 OMAHA: BMBF Förderkennzeichen 01ET0701/01ET0703
}

besondere der Frage nach, wie Multimorbidität, Autonomie und Lebensqualität in einem übergreifenden Rahmen konzipiert und mit standardisierten Messinstrumenten in der Allgemeinbevölkerung erfasst werden können. Dazu wurde ein populationsbasierter Zugang gewählt, um für die Gesundheits- und Sozialberichterstattung grundlegende Daten bereitzustellen, schwer zu erreichende Zielgruppen zu identifizieren, unterschiedliche Zugangswege $\mathrm{zu}$ überprüfen sowie Bedarfe für neue Zugangswege aufzuzeigen.

\section{Methoden}

Im Rahmen der OMAHA-Studie wurde eine nach Alter und Geschlecht stratifizierte Zufallsstichprobe von Personen ab 65 Jahren über das Einwohnermeldeamt (Berlin-Mitte, 15.07.2008) gezogen. Ausschlusskriterien waren: verstorben, außerhalb des Studiengebiets bzw. unbekannt verzogen oder Abwesenheit während der Studienzeit. Es fand ein schriftlicher Erstkontakt statt, dem ein in drei Gruppen randomisiertes Nachfassen zur Erhöhung der Teilnahmeraten folgte: (a) persönlicher Hausbesuch, (b) telefonischer Nachgang, (c) Erinnerungsschreiben. Auf der Grundlage standardisierter computergestützter persönlicher Interviews (CAPI) wurden mittels standardisierter Testverfahren insgesamt 31 (chronische) Gesundheitsprobleme sowie körperliche und kognitive Funktionseinschränkungen erfasst. Daneben wurden Daten zum subjektiven Wohlbefinden, psychischer Gesundheit und zur Lebensqualität erhoben. Ein Schwerpunkt lag in diesem Kontext auf der Validierung eines neuen Messinstruments für Lebensqualität bei Mehrfacherkrankungen (Fragebogen zur Lebensqualität multimorbider älterer Menschen, FLQM [51]). Ein standardisierter Selbstausfüllbogen ergänzte Fragen zu Soziodemografie und verschiedenen personalen und sozialen Ressourcen sowie möglichen Folgen von Multimorbidität (z. B. Behinderung in der Ausübung von Alltagsaktivitäten). Personen, die eine Teilnahme ablehnten, wurden gebeten, einen standardisierten Kurzfragebogen auszufüllen. Dieser diente unter anderem der Charakterisierung der NichtTeilnehmenden gegenüber den Teilnehmenden; zudem wurden die individuellen 
Gründe zur Nicht-Teilnahme erfragt. Alle Teilnehmenden der Ersterhebung werden längsschnittlich weiterverfolgt. Eine ausführliche Darstellung der Rekrutierungsund Erhebungsmethoden liefert das Studienprotokoll [49].

\section{Ergebnisse}

Nach Abzug der qualitätsneutralen Ausfälle (Wegzug, Tod, Abwesenheit während der Rekrutierungsphase) gingen von der Bruttostichprobe $(n=1.481)$ insgesamt 1.308 Personen in die Nettostichprobe ein. Trotz des erheblichen Rekrutierungsaufwands nahmen 299 Personen (22,9\% bezogen auf die Nettostichprobe) an der kompletten Ersterhebung teil. Durch ein stufenweises Vorgehen konnten die NichtTeilnehmer $(n=1.009)$ jedoch in folgende Gruppen aufgeteilt werden: (1) Personen, für die über einen Kurzfragebogen Eckdaten zum Gesundheits- und Funktionszustand erhoben werden konnten ( $\mathrm{n}=384,38,1 \%$ der Nicht-Teilnehmenden); (2) Personen, die eine Studienteilnahme komplett ablehnten $(n=324$; $32,1 \%)$; (3) Personen, die nie erreicht werden konnten $(n=301 ; 29,8 \%)$. Mindestens ein Ablehnungsgrund konnte von 86,6\% aller Nicht-Teilnehmer in Erfahrung gebracht werden. Von 299 Studienteilnehmern waren $97,32 \%(n=291)$ nach sechs Monaten zu einer zweiten Befragung bereit. Zum 18-Monats-Follow-up konnten 263 (88\%) der Studienteilnehmer befragt werden.

Die Studienteilnehmer (45,8\% Frauen) waren zum Zeitpunkt der Ersterhebung im Durchschnitt 75 Jahre alt. Es wurden durchschnittlich in vier Bereichen chronische Gesundheitsprobleme berichtet. Bluthochdruck, hohe Cholesterinwerte und Gelenkbeschwerden waren bei den Männern die am häufigsten genannten gesundheitlichen Probleme; bei den Frauen waren es Bluthochdruck, Gelenkbeschwerden und Rückenschmerzen [50]. Unterschiedliche Algorithmen zur Aggregation der Angaben zu den einzelnen Erkrankungen bzw. Bereichen gesundheitlicher Einschränkung besaßen einen unterschiedlichen prädiktiven Wert hinsichtlich gesundheitlicher Ereignisse im Längsschnitt: So konnten Sturzereignisse beispielsweise deutlich besser mittels Einbeziehung subjektiver Einschränkun- gen vorhergesagt werden als durch reine Bezugnahme auf die Anzahl betroffener Bereiche.

Analysen mit dem neuen Messinstrument FLQM [51] zeigten, dass er als Indikator für Lebenszufriedenheit im Alter auch bei Vorliegen von Mehrfacherkrankungen gut geeignet ist. Das Alter an sich spielte keine Rolle bei der Bewertung der eigenen Zufriedenheit. Personen, die sich durch den eigenen Gesundheitszustand in täglichen Aktivitäten eingeschränkt fühlten, berichteten jedoch zugleich eine niedrigere Zufriedenheit mit ihrem Leben als Teilnehmer ohne solche Einschränkungen [52].

In den meisten Fällen war es möglich, von den Nicht-Teilnehmern zumindest einen Grund für die Ablehnung zu erfahren, nur für knapp ein Drittel liegen jedoch Vergleichsdaten mit den Teilnehmenden vor. Die Einbeziehung von subjektiv erlebten Einschränkungen durch bestimmte Gesundheitsprobleme erwies sich längsschnittlich als guter Prädiktor für Gesundheitsereignisse; sie könnte daher in vielen Kontexten die Operationalisierung von Multimorbidität verbessern. Im Gegensatz zur reinen Zählung von Erkrankungen [49] spielten auf die Gesundheit insgesamt bezogene subjektive Einschränkungen auch in Bezug auf Lebenszufriedenheit eine entscheidende Rolle. Insgesamt sprechen die bisherigen Ergebnisse aus OMAHA dafür, bei der Beurteilung von Multimorbidität der Patientenperspektive bzw. der subjektiven funktionalen Ebene vermehrte Aufmerksamkeit zu schenken. Eine umfassendere Diskussion in Bezug auf die hier geschilderten Ergebnisse findet sich bei Holzhausen et al. [50].

\section{Demenz und Multimorbidität bei Älteren und Migranten (Projekt MIGRANT-DEM) $^{6}$}

\section{Ulrike Beinhoff, Matthias Riepe}

Auf dem Kontinuum von funktionaler Gesundheit und Pflegebedürftigkeit (• Abb. 1) lässt sich das Projekt MIGRANT-DEM (Demenz und Multimorbi-

\footnotetext{
6 MIGRANT-DEM: BMBF Förderkennzeichen 01ET0701
}

dität bei Migranten mit nicht-deutscher Muttersprache im urbanen Raum) der Erkennung der frühen Beeinträchtigung der funktionalen Gesundheit zuordnen. MIGRANT-DEM beschäftigte sich mit Verfahren zur Früherkennung demenzieller Erkrankungen und von Multimorbidität, den Auswirkungen dieser Syndrome auf Autonomie und Lebensqualität sowie der Analyse von Barrieren bei der Nutzung der Angebote des Gesundheitssystems für ältere Menschen mit Migrationshintergrund. Dabei sollten insbesondere sprachunabhängige Tests zur Diagnose von kognitiven Beeinträchtigungen und demenziellen Erkrankungen entwickelt werden.

\section{Methoden}

Im Rahmen der MIGRANT-DEM-Studie sollten vor allem ältere, mehrfach erkrankte Menschen mit Migrationshintergrund untersucht werden. Wie die Ergebnisse beispielsweise der OMAHA-Studie (s. 2.1) zeigen, sind ältere Menschen mit Migrationshintergrund schwieriger für wissenschaftliche Studien zu erreichen als Menschen ohne Migrationshintergrund. Die MIGRANT-DEM-Studie setzte daher auf die Ankündigung der Studie in russisch- und türkischsprachigen Zeitschriften und auf Informationsveranstaltungen im Rahmen von Kulturvereinen sowie auf die Kooperation mit Ärzten, die bereits in der medizinischen Betreuung von Personen mit russisch- und türkischsprachigem Migrationshintergrund aktiv waren. Personen mit russisch- und türkischsprachigem Migrationshintergrund wurden ausgewählt, da diese unter älteren Migranten in Berlin die größte Gruppe von Migranten repräsentieren [86].

International etablierte Screeningverfahren und neuropsychologische Tests zur Frühdiagnostik von Demenzerkrankungen, z. B. der Mini-Mental-Status-Test [28], der Memory-Impairment-Screen [16] und der California-Verbal-LearningTest [19], sowie standardisierte Testverfahren zur Erfassung depressiver Symptome, z. B. die Geriatrische Depressionsskala, wurden unter Berücksichtigung der etablierten Empfehlungen zur Übersetzung diagnostischer Instrumente ins Türkische und Russische übersetzt. Darüber hinaus wurden in Vorversuchen und ite- 


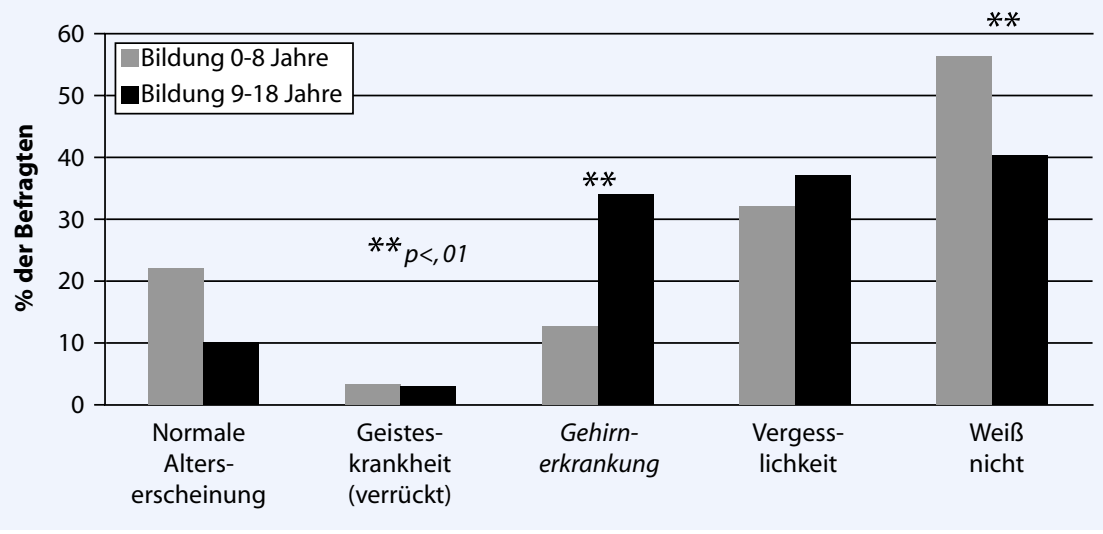

Abb. 3 A Unterschiede in der Konzeption von Demenz in Abhängigkeit von Bildung

rativ während der Probanden- und $\mathrm{Pa}$ tientenuntersuchung Methoden zur Abschätzung der generellen intellektuellen Befähigung und Verfahren zur spracharmen Testung episodischer Gedächtnisleistungen entwickelt. Es wurden 79 Probanden mit russischsprachigem Migrationshintergrund und 73 Probanden mit türkischsprachigem Migrationshintergrund untersucht. An einer weiteren Teilkohorte $(n=97)$ wurden außerdem Daten zum Krankheitswissen und Fragen zur Nutzung der Angebote des Gesundheitssystems erhoben.

\section{Ergebnisse}

Bei der Auswertung von Daten zur Inanspruchnahme medizinischer Versorgungsleistungen zeigte sich, dass ältere Migranten weniger Arztbesuche im Jahr berichten als z. B. vergleichbare deutsche Populationen. Während in der Gruppe älterer Migranten 30\% (russische Gruppe) bzw. 42\% (türkische Gruppe) angaben, dass ihr letzter Arztbesuch vier Monate oder länger zurücklag, waren dies in der Population der OMAHA-Studie nur 9,8\%. Auf die Frage, wie wichtig die Muttersprache des Arztes sei, antworteten 50\% der befragten Frauen und 66\% der befragten Männer, dass die Muttersprache des Arztes keine Rolle spiele. 97\% der Männer und $65 \%$ der Frauen gaben an, dass das Geschlecht des Arztes bei der Arztwahl keine Rolle spiele. Insgesamt fühlten sich $69 \%$ der männlichen und $54 \%$ der weiblichen Befragten in Bezug auf Krankheiten sehr gut oder gut versorgt und nur ein Bruchteil $(<5 \%)$ schlecht oder sehr schlecht versorgt. Dies deutet darauf hin, dass es weniger strukturelle Probleme der Inanspruch- nahme medizinischer Leistungen gibt als möglicherweise vielmehr Unterschiede in der Konzeption von Krankheiten wie der Demenz [71]. Ein Konzept von Demenz als Vergesslichkeit hatten lediglich 38\% bzw. 36\% der männlichen bzw. weiblichen Probanden mit türkischsprachigem Migrationshintergrund, und lediglich 45\% bzw. 16\% vertraten das Konzept einer Gehirnerkrankung. Solche Unterschiede in der Konzeption können an individuellen Ressourcen wie Bildung liegen. Es zeigte sich, dass signifikant mehr Personen mit höherer Bildung Demenz (korrekterweise) als Gehirnerkrankung konzipierten und gleichzeitig weniger oft der Kategorie „weiß nicht“ zustimmten (• Abb. 3).

Dieser Befund weist darauf hin, dass individuelle Ressourcen wie Bildung entscheidende Bedeutung für die Konzeption von Erkrankungen haben. Weil sich solche Konzeptionen auf die Inanspruchnahme von Versorgungsleistungen auswirken können, ist es bei Populationen wie älteren Migranten und deren Angehörigen besonders wichtig, auf adäquate Konzeptionen von Erkrankungen hinzuarbeiten, um ihnen im Angesicht von zunehmender Krankheitsbelastung auch durch demenzielle Erkrankungen ein Leben in hoher Lebensqualität und Autonomie zu ermöglichen [71].

Eine Voraussetzung, um dieses Ziel zu erreichen, ist die Entwicklung kulturadäquater Testverfahren. Aus der Analyse großer Zahlen von Patienten ist bekannt, dass die Einzelitems im Mini-Mental-Status-Test (MMSE) mit beginnender Alzheimererkrankung einen typischen Verlauf des Abbaus zeigen [1]. Es wurde eine Itemanalyse in den Probandenko- horten gerechnet, um zu prüfen, bei welchen Fragen Punktverluste zu verzeichnen sind. Eine Diskrepanz der Wahrscheinlichkeit, die Items richtig zu beantworten, zeigte sich insbesondere in Bezug auf instrumentelle Fähigkeiten wie Zeichnen, Schreiben und Rechnen; z. B. waren bei fast vollständiger räumlicher Orientierung nur circa 60\% der Probanden mit türkischsprachigem Migrationshintergrund in der Lage, die visuokonstruktive Aufgabe im MMSE zu lösen. In Bezug auf die spracharme und sprachgebundene Testung wurden im Rahmen der Studie Normwerte erhoben - auch hierbei zeigte sich, dass gerade in dieser Kohorte Bildung eine wesentliche konfundierende Variable der diagnostischen Schärfe der Instrumente zur Demenzfrüherkennung ist.

Insgesamt müssen die diagnostischen Instrumente zur Diagnose kognitiver Beeinträchtigungen bei Personen mit Migrationshintergrund unter Berücksichtigung konfundierender Variablen (z. B. Sprache, Bildung, instrumentelle Fähigkeiten) weiter verbessert werden. Um zukünftig Barrieren bei diagnostischen Instrumenten noch besser zu überwinden, wäre neben der Weiterentwicklung spracharmer Testverfahren der Einsatz neuer Technologien zu prüfen, wie etwa die Anwendung eines Spracherkennungssystems, welches die computergestützte Registrierung von Wörtern ermöglichen könnte.

\section{Ressourcen für Autonomie und Lebensqualität auf der Ebene des Individuums und des sozialräumlichen Kontextes (Projekt NEIGHBOURHOOD) $^{7}$}

\section{Katrin Falk, Josefine Heusinger, Kerstin Kammerer, Meggi Khan-Zvorničanin, Susanne Kümpers, Michael Zander}

Auf dem Kontinuum von funktionaler Gesundheit und Pflegebedarf lässt sich das Projekt NEIGHBOURHOOD (Autonomieerhalt angesichts von Sturzfolgen in sozial benachteiligten Quartieren und Nachbarschaften) im Bereich der pflegebedürftigkeitsbedingten Ab-

\footnotetext{
7 NEIGHBOURHOOD: BMBF Förderkennzeichen - 01ET0705/01ET0706
} 


\begin{tabular}{|c|c|c|c|}
\hline & $\begin{array}{l}\text { Innerstädtischer Bezirk } \\
\text { (Berlin-Moabit) }\end{array}$ & $\begin{array}{l}\text { Urbanes Neubaugebiet } \\
\text { (Berlin-Marzahn) }\end{array}$ & $\begin{array}{l}\text { Ländliche Region } \\
\text { (Beeskow im Landkreis Oder-Spree) }\end{array}$ \\
\hline Einkauf & $\begin{array}{l}\text { Selbständig möglich, überwiegend } \\
\text { zu Fuß, kaum Fahrtkosten, viele klei- } \\
\text { ne Läden vor Ort (täglicher Bedarf) }\end{array}$ & $\begin{array}{l}\text { Selbständig möglich, überwiegend zu } \\
\text { Fuß, geringe Fahrtkosten, überwiegend } \\
\text { beim Discounter }\end{array}$ & $\begin{array}{l}\text { In Dörfern Einkauf nur durch wöchentliche } \\
\text { mobile Geschäfte möglich, ansonsten durch } \\
\text { Nachbarn in der Stadt oder mit hohen Trans- } \\
\text { portkosten }\end{array}$ \\
\hline $\begin{array}{l}\text { Medizinische } \\
\text { Versorgung }\end{array}$ & Selbständig möglich, teilweise Taxi & $\begin{array}{l}\text { Selbständig möglich, teilweise zu Fuß, } \\
\text { Facharzt durch ÖPNV oder Taxi }\end{array}$ & Nur mit Hilfe möglich, hohe Transportkosten \\
\hline Soziale Partizipation & $\begin{array}{l}\text { Selbständig möglich, manchmal im } \\
\text { Haus, oft gar nicht }\end{array}$ & $\begin{array}{l}\text { Selbständig möglich, überwiegend } \\
\text { zu Fuß oder ÖPNV, Begleitung durch } \\
\text { Nachbarinnen und Nachbarn, teilweise } \\
\text { Mobildienste }\end{array}$ & $\begin{array}{l}\text { Seltene Angebote vor Ort, kaum selbständig } \\
\text { möglich, hohe Transportkosten }\end{array}$ \\
\hline
\end{tabular}

hängigkeit von Hilfen bei Menschen in Privathaushalten anordnen (• Abb. 1). NEIGHBOURHOOD ging den Fragen nach, wie individuelle Ressourcen an ökonomischem, kulturellem und sozialem Kapital [12] mit Ressourcen auf der Ebene der räumlichen und sozialen Umgebung interagieren. Dazu wurden drei qualitative Fallstudien im Raum BerlinBrandenburg realisiert. Für die Untersuchung wurde auf die neo-institutionalistische Theorie, die Theorie der sozialen Praxis [11] und Konzepte der Sozialraumanalyse zurückgegriffen.

\section{Methoden}

Im Rahmen der NEIGHBOURHOODStudie wurden drei exemplarisch ausgewählte Nachbarschaften im Berlin-Brandenburger Raum untersucht: das innerstädtische Altbauquartier Berlin-Moabit, das sich vor allem durch dichte Bebauung aus der Gründerzeit und einen hohen migrantischen Bevölkerungsanteil auszeichnet, die städtische Großwohnsiedlung Berlin-Marzahn und die ländliche Region Beeskow im Landkreis Oder-Spree. Alle drei Gebiete sind durch einen insgesamt niedrigen sozioökonomischen Status ihrer Bevölkerung geprägt.

Ziel der Fallstudien war es, die sozialräumliche Perspektive (Ebene der räumlich-technischen Umwelt, der sozialen Umwelt, Infrastruktur und Versorgung) mit der Ebene des Individuums (hier vor allem gender- und milieuspezifisch geprägte Ressourcen) in Verbindung $\mathrm{zu}$ bringen und ihre Interaktionen $\mathrm{zu}$ analysieren. Der Feldzugang wurde dabei so gewählt, dass über geeignete Multiplikatoren auch ältere Menschen erreicht wurden, die bislang noch keinen Zugang zu
Versorgungsleistungen und Hilfsangeboten hatten.

In Berlin-Moabit wurden 24 Menschen über 6o Jahre mit Unterstützungsund/oder Pflegebedarf für die Fallstudie interviewt, darunter sechs mit Migrationshintergrund. Auf der Ebene des Sozialraums wurden 24 Interviews mit Akteuren der Versorgung älterer Menschen in Moabit geführt (Akteure aus Lokalpolitik, Tagespflegeeinrichtungen, ambulanten Pflegediensten, Nachbarschaftshilfeeinrichtungen) und öffentlich zugängliche Dokumente dieser Akteure ausgewertet. Im Bezirk Marzahn wurden 20 ältere Menschen mit Mehrfacherkrankungen befragt und 24 Interviews mit Akteuren der Versorgung realisiert. Im Landkreis Oder-Spree wurden 20 Personen im Alter über 6o Jahre mit akutem Unterstützungs- und/oder Pflegebedarf in die Studie aufgenommen. Außerdem wurden 22 Interviews mit Akteuren der Versorgung älterer Menschen im Landkreis durchgeführt und Dokumente dieser Akteure untersucht.

Alle Fallstudien wurden mit qualitativen Methoden (Dokumentenanalyse, leitfadengestützte Interviews, teilnehmende Beobachtungen) untersucht. Für die Analyse der Daten wurde auf Ansätze der Grounded Theory [43], der Institutional Ethnography [85] und der qualitativen Netzwerkanalyse zurückgegriffen.

\section{Ergebnisse}

Zentrale Befunde des NEIGHBOURHOOD-Projekts zeigen, dass sozial benachteiligte Ältere vor allem über ihre sozialen Netzwerke und ihnen bekannte, aber nicht pflegespezifische professionelle Angebote Zugang zu Hilfen suchten.
Insbesondere Mobilitätseinschränkungen, fehlende Information über Unterstützungsangebote und (dadurch mit bedingt) der Mangel an sozialer Partizipation wurden von den Teilnehmern in allen drei Fallstudien als Hindernisse für Autonomie und Lebensqualität benannt. Eine umfassendere Diskussion der Ergebnisse findet sich in Heusinger et al. [48].

Die Ressourcen für die Deckung der Bedarfe des täglichen Lebens und für soziale Partizipation waren hingegen in den drei untersuchten Regionen ungleich verteilt. Dies wirkt sich auf Autonomiechancen und Wohlbefinden der Teilnehmer aus (• Tab. 3).

Der Zugang zu professionellen Hilfsangeboten gelang vielen befragten älteren Menschen erst nach einem Akutereignis wie einem Sturz und dem dadurch bedingten Krankenhausaufenthalt. Präventive Angebote z. B. zur Sturzprophylaxe gab es kaum. Vor allem isoliert lebende ältere Menschen und Menschen aus Migrantencommunities verfügten oft nicht über die notwendigen Informationen, um Hilfsangebote in der Nachbarschaft und im Quartier in Anspruch zu nehmen.

Individuelle Unterschiede im Umgang und in der Anpassung an die durch Multimorbidität im Alter bedingten Einschränkungen lassen sich mit der Ressourcenausstattung in Zusammenhang bringen: Insbesondere ältere Menschen, die über viel soziales, kulturelles und ökonomisches Kapital [12] verfügen, berichten trotz Einschränkungen über höhere Lebensqualität und Autonomie. Ihnen fiel es leichter, ihre Vorstellungen gegenüber Pflegediensten durchzusetzen und sie konnten mehr Ressourcen in Mobilität außerhalb des Hauses und in soziale Parti- 
zipation investieren. Vor allem im ländlichen Raum waren Partizipationsmöglichkeiten - kulturelle und soziale Angebote - ohnehin seltener, und individuelle Mobilitätseinschränkungen und räumliche Barrieren machten es älteren Menschen schwer, diese in Anspruch zu nehmen.

Diese Befunde auf der Ebene der Individuen wurden in den Interviews mit den Akteuren der Versorgung älterer Menschen bestätigt. Auf dieser Ebene zeigte sich zudem, dass an der Schnittstelle von Pflege und sozialer Teilhabe Zuständigkeits-, Informations- und Kooperationslücken zwischen Pflegeleistungserbringern und offener Altenhilfe bestehen und zudem Refinanzierungsmöglichkeiten für die Sicherung wie z. B. der Mobilität fehlen. Der Vergleich der Fallstudien belegte, dass eine aktive Vernetzung Potenziale erschließen kann und Synergien erlaubt, die bis in die Pflegehaushalte hinein nachweisbar sind. Regionale Unterschiede lassen sich sowohl mit mangelnden Ressourcen (Qualifikation, finanzielle Mittel) als auch unterschiedlichen politischen Prioritätensetzungen erklären.

\section{Schlafstörungen und \\ Multimorbidität in der Wechselwirkung (Projekt INSOMNIA) ${ }^{8}$}

\section{Uwe Flick, Vjenka Garms-Homolová, Joachim Kuck, Silke Migala, Gundula Röhnsch, Katrin Theiss}

Im Projekt INSOMNIA (Interrelation of Sleep Disorders and Multimorbidity in Nursing Institutions for the Aged) wurden Störungen des Schlafs und des WachSchlaf-Rhythmus bei mehrfach erkrankten älteren Menschen in Pflegeheimen untersucht. Die Forscher gingen der Frage nach, ob die Schlaf-Wach-Probleme nicht die Faktoren repräsentieren, welche die Entwicklung von funktionaler Abhängigkeit, damit auch den Bedarf an Hilfe und Pflege begünstigen [35]. In der schematischen Darstellung ( $\bullet$ Abb. 1) kann dieses Projekt weit rechts angesiedelt werden.

In der INSOMNIA-Studie wird aufgezeigt, dass bestimmte Schlafstörungen

8 INSOMNIA: BMBF Förderkennzeichen: 01ET0707 mit defizitären Ressourcen einhergehen, jedoch selber auch ein Risiko für die Faktoren darstellen [26], die normalerweise als „Ressourcen“ fungieren, und dass die Institution Pflegeheim auf Schlafstörungen, insbesondere jedoch auf die Beeinträchtigung des Wach-Kreislaufs, nicht mit der zur Verfügungstellung angemessener Ressourcen reagiert, sondern eher mit einer Ressourcenlimitierung [27, 35]. Der Fokus der Untersuchung lag sowohl auf Daten des individuellen Zustands der Bewohner als auch auf Vertretern pflegender Institutionen und strukturellen Merkmalen dieser Langzeiteinrichtungen [26].

\section{Methoden}

Der methodische Zugang des INSOMNIA-Projekts erforderte die Kombination mehrerer Datenquellen [26]. Der Zustand und die Situation von Heimbewohnern [27] wurde mit dem Minimum Data Set (MDS) des Resident Assessment Instruments 2.0 ([65], deutsche Fassung; [38]) erfasst. Diese Daten werden routinemäßig einmal pro Jahr zu Controllingszwecken der Krankenkassen erhoben. Das MDS des RAI 2.o enthält rund 320 Variablen, darunter demografische Angaben, Items zur Erfassung des kognitiven Zustands, kommunikative Fähigkeiten, Stimmungslage und Verhalten, psychosoziales Wohlbefinden, Activity of Daily Living, Angaben zu Bewegungsmodi und weitere Items der Funktionsfähigkeit, Kontinenz, zwei Sets von Diagnosen (erfasst einerseits von Pflegekräften, andererseits von behandelnden Ärzten), allgemeiner Gesundheitszustand, Ernährungsstatus, Beschäftigungsmuster, Medikation, Pflege und Therapien. Analysiert wurden drei Querschnittsstichproben aus drei aufeinander folgenden Jahren, insgesamt 7.505 Datensätze. Der Anteil von Frauen lag bei 71,5\%, und das Durchschnittsalter betrug 79,7 Jahre $(\mathrm{SD}=13,80)$. Im Schnitt wurden 4,9 Diagnosegruppen festgestellt. Die Pflegestufen verteilten sich folgendermaßen: keine Pflegestufe $(6,4 \%)$, Stufe $1(31,4 \%)$, Stufe $2(36,7 \%)$, Stufe $3(23,5 \%)$. Knapp die Hälfte der Teilnehmerinnen und Teilnehmer (46\%) litten unter mittleren bis sehr schweren kognitiven Beeinträchtigungen. Von 1.38o Bewohnern, welche die gesamte Laufzeit der Studie überlebt haben, konn- ten longitudinale Daten analysiert werden. Die Stichprobe entspricht in der Zusammensetzung großenteils der Verteilung von Pflegeheimbewohnerinnen und -bewohnern in Deutschland [87] und kann daher als repräsentativ angesehen werden. Von $80 \%$ der Bewohner (Versicherten der AOK Berlin-Brandenburg) konnten zudem sämtliche ärztliche Verordnungsdaten untersucht werden.

Die zweite Annäherung an die Problematik boten episodische Interviews mit Professionellen [26]. Befragt wurden 32 Pflegekräfte (7 Männer, 25 Frauen) teils aus Einrichtungen, aus denen auch die Assessment-Daten stammten, ferner 20 behandelnde Ärzte. Das subjektive Erleben von Schlafstörungen bei Bewohnerinnen $(\mathrm{n}=30)$ und Angehörigen $(\mathrm{n}=11)$ wurde ebenfalls mithilfe episodischer Interviews erhoben $[45,75]$. Schließlich dienten Begehungen von 16 Langzeitpflegeeinrichtungen zur Erhebung und nachfolgender Analyse der räumlich-strukturellen und organisatorischen Begebenheiten der Unterbringung und Pflege [61].

\section{Ergebnisse}

Entsprechend der Integration verschiedener methodologischer Konzepte konnten Ergebnisse gewonnen werden, die mehrere Betrachtungsebenen integrieren und die Aussagen des jeweils einen Zugangs aus weiteren Perspektiven verifizieren. Eine umfassendere Darstellung und Diskussion der Ergebnisse werden in einer Monographie [36] und zahlreichen weiteren, entweder bereits veröffentlichten oder in der Vorbereitung befindlichen Publikationen präsentiert (z. B. $[27,37,62$, 63]).

Auf der Ebene der Bewohner wurden die Prädiktoren von Schlafstörungen in autonomierelevanten Funktionsbereichen untersucht. Dazu wurden standardisierte Differenzen vom Skalenmittelwerten verwendet, vor allem wurden der ADL-Index (Aktivitäten des täglichen Lebens; [67]), Kommunikationsfähigkeit (gemessen mit Kommunikationsskala/sCom; [32]), Schmerzen (Pain Scale; [34]), Depression (Depression Rating Scale/DRS; [15]), soziales Engagement (mit dem Index of Social Engagement/ISE; [41, 64]) und Konflikte im Heim (additive Skala [36]) miteinander verglichen (• Abb. 4). 
Dabei zeigte sich beispielsweise für die Insomnie, dass Personen, bei denen dieses Problem täglich auftritt, in allen genannten Funktionsbereichen über weniger Ressourcen verfügten. Prognostisch betrachtet, erweist sich die Insomnie als ein signifikanter Prädiktor der fortschreitenden Reduktion von einzelnen Fähigkeiten (gemessen im Zeitraum von 90 Tagen), aber auch der Gesamtverschlechterung des Funktionsstatus [40]. Im Querschnitt betrachtet hatten Personen, bei denen sich diese Form der Schlafstörung nicht manifestierte, eine fünffach höhere Chance, keine Beeinträchtigungen der kognitiven Leistungsfähigkeiten (gemessen mit dem Cognitive Perfomance Score [66]) zu erleiden ( $\mathrm{OR}=5,19,95 \%$ $\mathrm{CI}=2,45-11$ ), eine doppelt so hohe Chance, in ihren Aktivitäten des alltäglichen Lebens unabhängig $\mathrm{zu}$ sein $(\mathrm{OR}=1,97$; $95 \% \mathrm{CI}=1,11-3,56)$ und über intakte Kommunikation zu verfügen $(\mathrm{OR}=2,07$; 95\% CI = 1,11-3,36).

Die Analyse der Interviews mit Pflegemitarbeitern ergab ein geringes Problembewusstsein und fehlende Kenntnisse über den Schlaf, Schlaf-Wach-Rhythmus und deren Störungen. Die Deutungs- und Handlungsmuster, das heißt die aus dem Interviewmaterial erarbeiteten „subjektiven Theorien“ der Pflegenden hinsichtlich der Relevanz des normalen und gestörten Schlafs und Wachens, zeigten, dass die Mehrheit dazu tendiert, die pflegerische oder präventive Handlungserfordernis zu verneinen. Nur die Begründungen unterscheiden sich je nach Grad der professionellen Kompetenz. Während sich die einen Mitarbeiter auf die Selbstbestimmung und lebenslangen Gewohnheiten der Bewohner beriefen, die es in jedem Falle zu respektieren gilt, sodass die Motivierung der Bewohner ihre Schlafgewohnheiten zu verändern nicht in Frage kam, ignorierten die anderen Mitarbeiter die pflegerische Zuständigkeit für das Schlafen oder Wachen. Eine „schonende Haltung“ angesichts der Schlafprobleme, insbesondere das Hinnehmen der exzessiven Schläfrigkeit, fanden ihre Entsprechung in den Ergebnissen der Assessmentdaten [26]. Gerade diese Störung scheint große Barrieren im Zugang zu institutionellen Ressourcen zu induzieren [39].

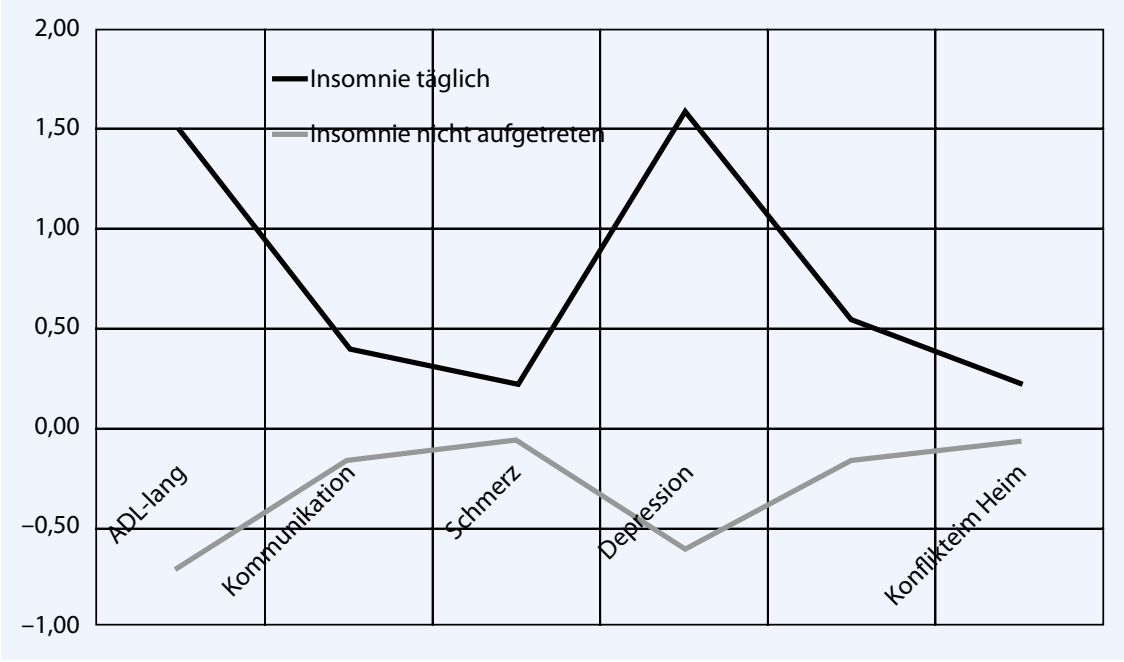

Abb. 4 A Prädiktoren von Schlafstörungen bei $n=2.577$ Heimbewohnern. Höhere Werte = stärkere Beeinträchtigungen. Alle Mittelwertunterschiede sind signifikant $(p<0,001)$

Der übermäßige Schlaf und die lange im Bett verbrachte Zeit, die - wie die Untersuchung zeigte - mit Risiken für die verbleibende Gesundheit und Selbstversorgungsfähigkeit behaftet sind, stellen somit die Probleme dar, die genau so viel oder sogar mehr Aufmerksamkeit verdienen, wie die Insomnie [39]. Doch gerade sie finden im institutionellen Kontext der Pflegeheime zu wenig Beachtung. Nach wie vor fanden sich „Nachtzeiten“ von 12 bis 16 Stunden, weil abendliche Beschäftigungsmöglichkeiten fehlten, die Bewohner bereits um 18 Uhr ins Bett gebracht wurden und am Morgen niemand dabei war, der rechtzeitig aus dem Bett geholfen hätte. Auch die Licht- und Lärmverhältnisse waren in vielen Einrichtungen nicht für angemessene Schlafqualität und Schlafdauer günstig. Nur in $8,6 \%$ der Einrichtungen war die Tages-/Nachbeleuchtung so beschaffen, dass der Schlaf-WachRhythmus der Bewohner unterstützt wurde. Obwohl der Zusammenhang zwischen nächtlichem Lärm und Schlafstörungen in den betroffenen Einrichtungen bekannt war, wurde keine Abhilfe vorgesehen. Regelmäßige Aufenthalte im Freien ließen sich für die überwiegend bettlägerigen Personen in den wenigsten Einrichtungen realisieren.

Insgesamt zeigt die INSOMNIA-Studie einerseits, dass Personen mit bestimmten Formen von Schlafstörungen von vornherein reduzierte Ressourcen mitbringen. Andererseits erschweren diese Schlafstörungen ebenfalls die Erhaltung der indi- viduellen Ressourcen der Bewohner, aber auch den Zugang zu externen Ressourcen, die in den Einrichtungen der Langzeitpflege vorhanden sind oder sein sollten [35]. Die funktionalen Konsequenzen von Multimorbidität werden von Schlafstörungen signifikant verstärkt [35].

\section{Schmerzen und Autonomie in Pflegeheimen (Projekt PAIN) ${ }^{9}$}

\section{Dagmar Dräger, Sebastian Ellert, Sonja Kalinowski, Marita Kölzsch, Reinhold Kreutz, Ines Wulff}

Im Projekt PAIN („Pain and Autonomy in the Nursing Home") wird das Phänomen Schmerz bei mehrfach erkrankten Bewohnern in Pflegeheimen vor dem Hintergrund von Autonomie näher untersucht. Damit liegt das Projekt, ähnlich wie das Projekt INSOMNIA, eher auf der rechten Seite des Kontinuums von funktionaler Gesundheit und Pflegebedürftigkeit (• Abb. 1).

Mit zunehmendem Alter steigt die Prävalenz vor allem chronischer Schmerzen stark an. Internationale Studien gehen davon aus, dass zwischen $49 \%$ und $86 \%$ der Pflegeheimbewohner unter Schmerzen leiden [70]. Schmerzen und insbesondere die Erwartung von Schmerzen können dazu führen, dass ältere Menschen Bewegungen gezielt vermeiden $[58,59]$ und so in ihrer Autonomie über die Schmerzen

9 PAIN: BMBF Förderkennzeichen - 01ET0701 


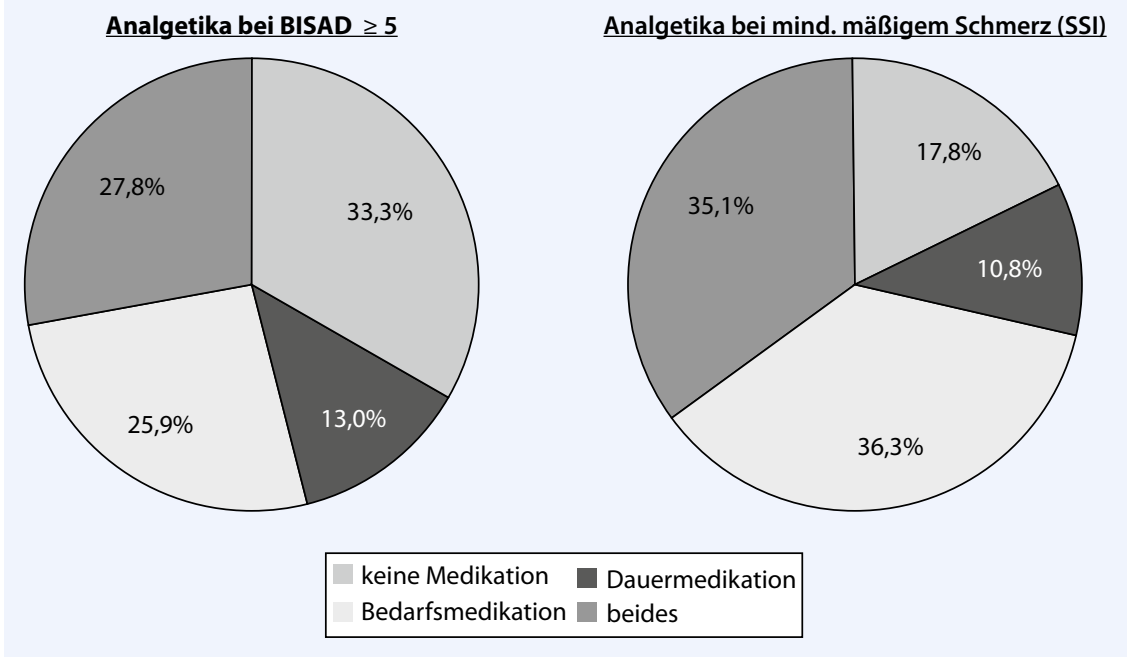

Abb. $5 \Delta$ Verordnungshäufigkeit von Analgetika bei Bewohnern mit deutlichem Schmerzverhalten mit schweren kognitiven Einschränkungen nach Beobachtung mit BISAD $(n=54)$ im Vergleich zu Personen mit mindestens mäßigen Schmerzen nach Interview mit SSI $(n=157)$

hinaus durch Einschränkungen in ihrem funktionalen Status beeinträchtigt werden.

Um Ressourcen für eine adäquate Schmerzversorgung in Pflegeheimen zu untersuchen, wurde im Projekt PAIN eine Untersuchung über mehrere Ebenen realisiert - neben Daten zum Schmerzerleben, zur medikamentösen Versorgung und Autonomie auf der Ebene der Bewohner wurden auch Daten auf der Ebene der Pflegeheime erhoben, wie z. B. im Hinblick auf die personelle sowie strukturelle Heimausstattung und Angebote zur Bewegungsförderung.

\section{Methoden}

Die PAIN-Studie wurde in einer $\mathrm{Zu}$ fallsauswahl von insgesamt 40 Pflegeheimen in Berlin und Brandenburg (aus einer Grundgesamtheit von 586 Heimen) durchgeführt. In diesen Heimen wurden wiederum $40 \%$ der Bewohner per $\mathrm{Zu}$ fall mit einem Oversampling von 3:2 zugunsten von Männern ausgewählt. Für die Untersuchung kamen standardisierte Fragebögen, strukturierte Beobachtungen und körperliche Assessments zum Einsatz. Schmerzen wurden in Abhängigkeit vom kognitiven Zustand der Teilnehmenden entweder mit dem Strukturierten Schmerzinterview (SSI; [7]) oder mit dem BISAD, einem Beobachtungsinstrument [25], erfasst. Die Angemessenheit der Schmerzmedikation wurde mit einer deutschen Adaptation der Pain Medica- tion Appropriateness Scale (PMAS; [53]) ermittelt. Eine übersetzte Version der Hertz Perceived Enactment of Autonomy Scale (HPEAS; [46]) ermöglichte die Messung der subjektiv wahrgenommenen Autonomie der Heimbewohner, während für die Ermittlung der Handlungsfähigkeit, als weiterer Aspekt der Autonomie, der Barthel-Index [6o] und der TimedUp-\&-Go-Test [69] zum Einsatz kamen.

Insgesamt wurden 560 Bewohner untersucht. Die Teilnehmenden waren im Schnitt 81,2 Jahre alt, 39\% waren Männer. Im Schnitt lagen 4,6 Diagnosegruppen vor. Die Pflegestufen verteilten sich folgendermaßen: keine Pflegestufe $(1,4 \%)$, Stufe $1(34,6 \%)$, Stufe $2(42,3 \%)$, Stufe 3 $(21,6 \%)$. Über ein Drittel der Teilnehmer $(37,3 \%)$ litten unter sehr starken kognitiven Beeinträchtigungen (MMSE $\leq 9)$. Die Stichprobe entsprach hinsichtlich der Verteilung der Pflegestufen annähernd der Zusammensetzung der Pflegeheimbewohner in Deutschland [87].

\section{Ergebnisse}

Deskriptive Analysen zeigten, dass 55,8\% der Personen mit einem MMSE $\geq 10$ Schmerzen angaben. In der Mehrzahl handelte es sich um ein chronisches Schmerzgeschehen, denn $64,3 \%$ dieser Bewohner litten seit Jahren unter diesen Beschwerden. Es wurde sowohl von dauerhaften Schmerzen (36,7\%) als auch über immer wieder auftretende Schmerzattacken $(59,2 \%)$ berichtet. In der Intensi- tät beschrieben $66,8 \%$ der befragten Personen ihre Schmerzen als "mäßig" oder „stark“, 13,3\% klassifizierten ihre Schmerzen als „sehr stark“ oder "unerträglich“. Die in dieser Teilstichprobe als zweithäufigste Diagnose ermittelten Erkrankungen des Bewegungsapparates spiegeln sich in den genannten schmerzhaften Körperstellen wider. Die im Durchschnitt an vier unterschiedlichen Körperstellen auftretenden Schmerzen, ohne dass ein bestimmter Schmerzort im Mittelpunkt stand, verweisen deutlich auf eine Verbindung zur Multimorbidität. Ein umfangreiches Morbiditätsspektrum (Cumulative Illness Rating Scale [CIRS] >6 Punkten) verdoppelt das Risiko an Schmerzen zu leiden $(2,0 \mathrm{KI} 1,2-3,5)$. Ein erhöhtes Risiko für das Schmerzvorkommen konnte auch bei Bewohnern, die unter Depression/Niedergeschlagenheit litten, ermittelt werden $(2,2 \mathrm{KI} 1,3-3,8)$.

Von 202 Bewohnern mit schweren kognitiven Beeinträchtigungen (MMSE $\leq 9$ ) wurden bei $54,5 \%$ Hinweise auf schmerzbezogenes Verhalten ermittelt. Insgesamt stehen diese Befunde für eine relativ hohe Prävalenz von Schmerzerleben in Pflegeheimen. Eine umfassendere Diskussion der Ergebnisse findet sich in Dräger et al. [20].

Die Frage nach adäquater Schmerzmedikation wurde durch die PMAS erfasst. Hutt et al. [53] gehen davon aus, dass bei Anwendung dieses Instruments ein erzielter Wert von $\leq 67 \%$ auf eine unangemessene Schmerzmedikation hinweist.

In der untersuchten Stichprobe wurde bei den Bewohnern, die eine Therapie mit Analgetika bekamen, nur bei der Gruppe der Personen, die dauerhaft und zusätzlich bei Bedarf Schmerzmedikamente erhielten, eine adäquate Versorgung festgestellt. Insgesamt erhielten drei Viertel der Bewohner mit Schmerzen keine angemessene Schmerzmedikation. Weiterhin zeigte sich, dass $17,8 \%$ der Befragten mit mindestens mäßigen Schmerzen und 33,3\% der kognitiv stark beeinträchtigten Personen mit deutlichen Hinweisen auf Schmerzverhalten (BISAD $\geq 5$ ) überhaupt keine Schmerzmedikation erhielten (• Abb. 5).

Neben der medikamentösen Therapie ist auch die empfohlene Anwendung nicht-medikamentöser Maßnahmen [1, 
2, 23] unzureichend. Nur 23 Personen der insgesamt 306 Bewohner mit Schmerzen oder mit wenigstens einer schmerzbezogenen Verhaltensweise laut BISAD erhielten eine Schmerztherapie aus dem Bereich der peripher wirkenden Maßnahmen und der körperlichen Übungen. Bewohner mit starken kognitiven Beeinträchtigungen (MMSE $\leq 9$ ) waren erneut benachteiligt und erhielten in nur zwei Fällen Maßnahmen dieser Art. Der Einsatz kognitiv-verhaltensorientierter Verfahren, als wichtiger Bestandteil der Schmerztherapie, konnte bei keinem Bewohner ermittelt werden.

\section{Diskussion}

Die Ergebnisse der Studien aus dem Berliner Forschungsverbund „Autonomie trotz Multimorbidität im Alter“ (AMA) zeigen, dass Autonomie und Lebensqualität älterer Menschen mit Mehrfacherkrankungen auf verschiedenen Ebenen von Ressourcen abhängig sind. Diese Ressourcen sind sowohl innerhalb der Person (s. Projekte PREFER, OMAHA, MIGRANT-DEM und NEIGHBOURHOOD), in sozialen Beziehungen, im sozialräumlichen Umfeld (s. Projekt NEIGHBOURHOOD), in institutionellen Bezügen (s. Projekte INSOMNIA und PAIN) und in der Versorgungslandschaft (s. Projekte NEIGHBOURHOOD, MIGRANT-DEM) verortet.

Die Studien im AMA-Verbund zeichnen sich durch den Einbezug von Menschen in verschiedenen Lebenswelten aus und sind auf einer Achse angesiedelt, die von der (fast vollständigen) funktionalen Gesundheit bis hin zu Gebrechlichkeit mit einem hohen Bedarf an Hilfen und Pflege reicht. Damit spiegeln die Teilprojekte die Vielfalt des Älterwerdens wider [94] und werden so der Komplexität und Pluralität der gesundheitlichen Situation von Menschen mit Mehrfacherkrankungen gerecht.

Die Projekte orientierten sich in der Auswahl der Studienpopulationen und der untersuchten Ressourcen an einem hypothetischen Kontinuum von zunehmendem Pflegebedarf und abnehmender funktionaler Gesundheit (• Abb. 1). Vor diesem theoretischen Hintergrund wird ersichtlich, dass in verschiedenen Stadien funktionaler Gesundheit je unterschiedliche Ressourcenkonstellationen von Bedeutung sind, um Autonomie [47] und Lebensqualität [31] trotz Multimorbidität zu erhalten.

\section{Ressourcen für Autonomie und Lebensqualität auf mehreren Ebenen}

Auf der Ebene des Individuums haben sich vor allem Ressourcen, die die Personen zu Handlungen befähigen, als wichtig herausgestellt. Zum Beispiel sind Selbstwirksamkeitserwartungen [6] für körperliche Aktivität und damit für funktionale Gesundheit und Autonomie wichtig [92, 93] (Projekt PREFER). Solche Einstellungen und personale Ressourcen wie z. B. Bildung, soziales Netzwerk, Health Literacy werden auch als kulturelles oder soziales Kapital [12] konzipiert und ermöglichen es älteren Menschen, den Herausforderungen durch Multimorbidität in sozioökonomisch benachteiligten Gebieten adaptiv zu begegnen (Projekt NEIGHBOURHOOD; [48]). Sowohl für gesundheitsbezogene Einstellungen, aber auch für andere individuelle gesundheitsbezogene Ressourcen wie Altersbilder oder spezifisches Wissen über Versorgungsangebote und über Krankheitskonzepte ist Bildung eine wichtige individuelle Voraussetzung [4]. Im Projekt MIGRANT-DEM zeigte sich exemplarisch, wie sich Bildung auf die subjektiven Krankheitskonzepte von Demenz auswirkt: Unter den Personen, die angeben konnten, dass es sich bei Demenz um eine Gehirnerkrankung handelt, waren signifikant mehr Personen mit höherer Bildung [9]. Befunde aus dem Projekt NEIGHBOURHOOD [48] zeigten eine mögliche Wirkrichtung auf: Personen mit höherem sozialen, ökonomischen und kulturellen Kapital [12] wie etwa höherer Bildung können besser mit krankheitsbezogenen Einschränkungen im Alltag umgehen, indem sie beispielsweise eher von sozialräumlichen Ressourcen wie Versorgungsangeboten für ältere Menschen Gebrauch machen und so ihre Autonomie aufrecht erhalten können. Ob und inwiefern im Sozialraum solche Ressourcen vorhanden sind, hat ebenfalls einen wichtigen Ein- fluss auf Gesundheit, Lebensqualität und Autonomie [10].

Im Projekt OMAHA zeigte sich, dass subjektiv erlebte Einschränkungen aufgrund der eigenen Gesundheitssituation einen deutlichen Einfluss auf die Lebenszufriedenheit hatten [52]. Die Förderung und der Erhalt subjektiver Funktionsfähigkeit scheint ein entscheidender Faktor auch in Bezug auf objektive, gesundheitsrelevante Ereignisse zu sein [50].

$\mathrm{Zu}$ den institutionellen Ressourcen, die sich auf Lebensqualität und Autonomie auswirken, zählen vor allem die professionelle Kompetenz der Pflegemitarbeiter und die kompetente ärztliche Versorgung sowie ein förderndes Konzept, um dessen Umsetzung sich alle Professionellen konsequent bemühen [35]. Im Hinblick auf das untersuchte Problem der Schlafstörungen sind auch die Organisation der Einrichtung und deren Mikroumwelt wichtig. Im Projekt INSOMNIA zeigte sich, dass die Bewohner trotz ihrer Multimorbidität noch über vielfältige Ressourcen verfügten, die aber hochgradig vulnerabel und abhängig von der Bereitstellung der genannten institutionellen Ressourcen waren. Je mehr die individuellen Ressourcen gefährdet oder gar eingeschränkt waren, was bei Personen mit bestimmten Formen von Schlafstörungen der Fall ist, desto weniger gelingt auch die Nutzung der institutionellen Ressourcen, weil diese sich als limitiert erweisen.

Die Befunde des PAIN-Projekts [20] wiesen auf einen ähnlichen Ursachenkomplex hin. Eine problematische Verordnungspraxis von Medikamenten, die sich auch in relativ häufiger, potenziell inadäquater Medikation niederschlägt [55], weist auf Defizite in der Ausbildung und der Allokation von Ressourcen im institutionellen Ablauf hin. Individuelle Ressourcen wie guter körperlicher Funktionsstatus, Kommunikationsfähigkeit oder soziales Engagement schützen vor negativen gesundheitlichen Konsequenzen von Multimorbidität wie Schlaflosigkeit (s. oben und [36]), gleichzeitig deuten diese Befunde aber auch darauf hin, dass Menschen mit wenig individuellen Ressourcen besonders anfällig für Einbußen in Lebensqualität [30] und Autonomie [47] durch Multimorbidität sind. 
Diese Befunde zeigten, dass sich im Themenkomplex Multimorbidität im Alter zum einen die Vielfalt des Alterns widerspiegelt [94], andererseits aber auch, dass die negativen Konsequenzen von Multimorbidität durch Ressourcen auf mehreren Ebenen und durch die Interaktion von Ressourcen auf mehreren Ebenen bestimmt wird [79].

\section{Spezifische Ressourcen für Autonomie und Lebensqualität}

Neben der Lokalisation von Ressourcen für Autonomie und Lebensqualität auf verschiedenen Ebenen machen die Befunde der Studien des AMA-Verbundes aber auch deutlich, dass für Personen mit unterschiedlichen Voraussetzungen in Bezug auf funktionale Gesundheit und Pflegebedarf unterschiedliche Ressourcen wichtig sind [13]. Durch die Verortung der untersuchten Studienpopulationen auf unterschiedlichen Punkten eines Kontinuums von abnehmender funktionaler Gesundheit und zunehmendem Pflegebedarf (• Abb. 1) konnten wichtige Ressourcen für die jeweiligen Populationen identifiziert werden, die in geeigneten Maßnahmen gezielt gefördert werden können.

Personen mit hoher funktionaler Gesundheit und geringem Pflegebedarf, die auf dem Kontinuum ( $\bullet$ Abb. 1) eher links anzusiedeln sind, haben ein hohes Potenzial dafür, durch gezielte Förderung beispielsweise von körperlicher Aktivität [21] ihren hohen funktionalen Status aufrechtzuerhalten und so länger selbständig leben zu können. Die Befunde aus der PREFERStudie legen nahe, dass für körperliche Aktivität vor allem Selbstwirksamkeitserwartungen und soziale Unterstützung wichtig sind [93]. Diese Faktoren lassen sich durch geeignete Interventionsmaßnahmen [3] gezielt fördern. So kann über Veränderungen in individuellem Gesundheitsverhalten nachhaltig Gesundheit verbessert und Autonomie und Lebensqualität aufrecht erhalten werden [72].

Allerdings kann der Zugang zu älteren Menschen mit Mehrfacherkrankungen für solche gezielten Maßnahmen schwierig sein. Befunde des OMAHA-Projektes zeigen, dass vor allem die Personengruppen mit dem höchsten Bedarf für
Gesundheitsförderung am schwierigsten zu erreichen sind [50], was im Einklang mit bisheriger Forschung zur Erreichbarkeit von bestimmten Zielgruppen für Gesundheitsförderung steht (z. B. [68]).

Die Projekte NEIGHBOURHOOD und MIGRANT-DEM berichten übereinstimmend, dass Bildung eine wichtige Ressource für Autonomie und Gesundheit darstellt. Sowohl die Nutzung von Angeboten der Gesundheitsversorgung [48] als auch die Konzeption von Krankheiten [8] hängt von Bildung ab. Wenn Autonomie als das Vorhandensein von Wahlmöglichkeiten [76] konzipiert wird, wird auch deutlich, dass der Raum dieser Wahlmöglichkeiten von Bildung abhängt: Bildung ermöglicht es, mehr und möglicherweise bessere Handlungsalternativen wahrzunehmen. Wenn Bildung hauptsächlich als Bildungsstatus verstanden wird, sind die Veränderungsmöglichkeiten vor allem im höheren Alter eher gering; allerdings können die an Bildung gekoppelten Wissens- und Informationsressourcen durchaus in geeigneten Interventionsmaßnahmen, die genau auf die Zielgruppen zugeschnitten sind, verbessert werden. In der NEIGHBOURHOODStudie konnte dementsprechend gezeigt werden, wie schwer erreichbare Zielgruppen, u. a. mit geringer Bildung und/oder Migrationshintergrund, durch spezifisch auf sie zugeschnittene Angebote erreicht werden können [22].

Gleichzeitig weisen die Befunde des NEIGHBOURHOOD-Projektes darauf hin, dass sozialräumliche Ressourcen einen entscheidenden Einfluss auf die Autonomie und Lebensqualität älterer Menschen haben [48]. Diese Befunde replizieren zum einen frühere Forschung (z. B. [1o]), zeigen aber durch die Identifikation von bislang ungenutztem Vernetzungspotenzial auf der Ebene der Akteure in der Versorgung älterer Menschen eine konkrete Perspektive zur Verbesserung von Autonomie und Lebensqualität auf.

Für ältere Menschen in Pflegeheimen wurden sowohl individuelle Ressourcen identifiziert, die Schlafstörungen vorbeugen können, als auch Ressourcen auf der Ebene der Institutionen selbst, die für die Versorgung der Bewohnerinnen und Bewohner ausschlaggebend sind $[20,36]$. Während frühere Studien bele- gen, dass sich individuelle gesundheitsbezogene Ressourcen auch noch bis ins höchste Alter und bei großem Pflegebedarf verbessern lassen [24], sind institutionelle Voraussetzungen eher durch gezielte Maßnahmen zur gesundheitsbezogenen Unternehmensentwicklung zu verbessern, wie beispielsweise die Einführung einer interdisziplinären Handlungsempfehlung für ein adäquates Schmerzmanagement einschließlich konkreter Vorgaben hinsichtlich der Schmerzmedikation [55].

\section{Einschränkungen und Stärken}

Neben Einschränkungen, die für die einzelnen Studien des AMA-Verbundes spezifisch sind und durch Design und Machbarkeit bedingt sind, wie z. B. dem Fehlen von objektiven Verhaltensdaten in der PREFER-Studie, der niedrigen Teilnahmerate in der OMAHA-Studie, der Beschränkung auf türkische und russische Migranten in der MIGRANT-DEM-Studie, den kleinen Stichproben in der NEIGHBOURHOOD-Studie, die in der INSOMNIA-Studie nicht vorhandene Möglichkeit einer vielfältige Differenzialdiagnostik für Schlafstörungen und dem querschnittlichen Design der PAIN-Studie sind einige allgemeinere Punkte bei der Interpretation der Ergebnisse wichtig.

Die Verortung der einzelnen Studien auf dem hypothetischen Kontinuum von Pflegebedarf und funktionaler Gesundheit erfolgte nicht anhand einer Bedarfsanalyse an epidemiologischen Daten [42], sondern Theorie geleitet. Diese Herangehensweise hat den Vorteil, dass theoretisch die Vielfalt der Lebensformen im Alter $[27,94]$ abgebildet werden kann. Nachteilig ist, dass dieses Vorgehen den Tatbestand ausschließt, dass die untersuchten Populationen in unterschiedlichen Settings Ähnlichkeiten im Hinblick auf die Ausprägung der funktionalen Einschränkungen zeigten; sowohl in der Gemeinde, als auch in Pflegeinstitutionen fanden sich einerseits Menschen mit Pflegebedarf, andererseits relativ unabhängige alte Frauen und Männer.

Eine weitere Einschränkung besteht darin, dass die Studien kein einheitliches Erhebungsinstrument für Autonomie hatten. Während Lebensqualität in allen Stu- 
dien durch den EQ-5D [14] erfasst wurde, konnte für die Erfassung von Autonomie aufgrund der Vielfalt von Konzeptionen kein für alle Fragestellungen geeignetes Instrument identifiziert werden. Allerdings finden sich in allen Studien Daten zur Erfassung von allgemeinen und instrumentellen Aktivitäten des täglichen Lebens $[54,57]$, sodass zumindest vergleichende Analysen zur funktionalen Gesundheit als wichtige Voraussetzung von Selbständigkeit im Alter möglich sind.

Neben diesen Einschränkungen stellt die Vielfalt der untersuchten Ressourcen und die dadurch mögliche gezielte Förderung von Lebensqualität und Autonomie in klar definierten Subpopulationen eine dezidierte Stärke der Projekte des AMAVerbundes dar. Die Projekte untersuchten Menschen, die das gesamte Spektrum von Multimorbidität, von funktional gesunden, kaum eingeschränkten Menschen im Privathaushalt bis hin zu schwerst pflegebedürftigen und kognitiv eingeschränkten Menschen im Pflegeheim abbilden.

\section{Implikationen und Ausblick}

Die Studien des Berliner Forschungsverbundes AMA haben Ressourcen in verschiedenen Populationen mehrfach erkrankter älterer Menschen aufgezeigt. Diese Ressourcen umfassen ein Spektrum individueller, sozialer, sozialräumlicher sowie organisatorisch-institutioneller Einflussfaktoren auf den Kontext von Autonomie und Multimorbidität. Es konnte gezeigt werden, dass für Personen mit unterschiedlichem funktionalem Gesundheitsstatus jeweils unterschiedliche Ressourcenkonstellationen wichtig sind, um Autonomie und Lebensqualität zu erhalten. Solche Ressourcen lassen sich durch geeignete Maßnahmen gezielt fördern - das ist die Grundannahme für die weitere Arbeit des AMA-Verbundes.

In einer zweiten, durch das BMBF geförderten Studienphase (2011-2013) werden in Teilprojekten geeignete Maßnahmen der Ressourcenförderung entwickelt und evaluiert. Die in den Projekten des Verbundes geplanten Interventionen zielen auf die Mobilisierung personaler, sozialer, professioneller und institutioneller Ressourcen. Die Interventionen sollen so konzipiert werden, dass sie in der Pra- xis nachhaltig, zielgruppenorientiert und ethisch vertretbar eingesetzt werden können und zudem von der Zielgruppe älterer Menschen akzeptiert und nachhaltig in Anspruch genommen werden.

Ziel aller Interventionen ist der Erhalt von Autonomie älterer Personen trotz Multimorbidität. Damit trägt der Verbund auch weiterhin dazu bei, Eckpunkte einer modernen, die Autonomie trotz vorhandener Gesundheitseinbußen stützenden Umwelt-, Versorgungs- und Pflegepraxis als Strategien, Modelle und Programme zu entwerfen.

\section{Korrespondenzadressen \\ Prof. Dr. A. Kuhlmey}

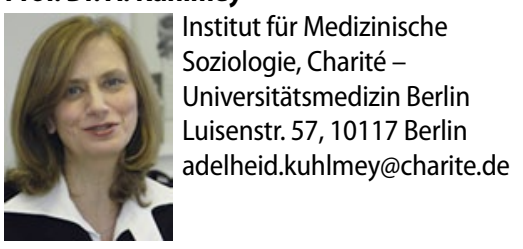

\section{Prof. Dr. C. Tesch-Römer}

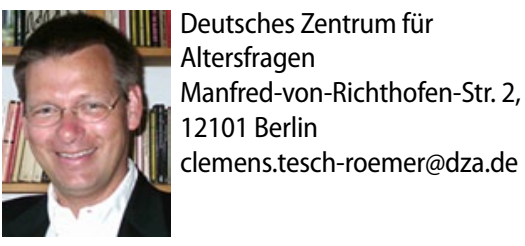

Danksagung. Der Forschungsverbund Autonomie trotz Multimorbidität im Alter" (AMA) bedankt sich beim Bundesministerium für Bildung und Forschung (BMBF) für die dreijährige Förderung und beim Projektträger Deutsches Zentrum für Luft- und Raumfahrt (DLR) für die stete Unterstützung und Begleitung der Projektarbeit.

Alle Verbundmitarbeiterinnen und -mitarbeiter fühlen sich dem wissenschaftlichen Beirat des Forschungsverbundes für seine konstruktive Begleitung verbunden. - Darüber hinaus gilt der Dank des PREFER-Projektes seinen Studienteilnehmerinnen und Studienteilnehmern. Zudem wird den Studentinnen und Studenten, die im Rahmen des Projektes gearbeitet und/oder ihre Qualifizierungsarbeiten angefertigt haben, gedankt: S. Lysk, C. Marx, J. Tholeikis und S. Weinz. - Das Projekt OMAHA sagt insbesondere den Kollegen Dr. J. Welke, Dr. U. Hapke und Dr. H. Knopf für die fachliche Kritik und Rückmeldung sowie für wesentliche Impulse bei der Ausgestaltung der Studie dankeschön. Für ihre unersetzliche Arbeit im Studiensekretariat wird I. Kurzawe-Seitz, für statistische Analysen und das verlässliche Datenmanagement wird A. Ernert gedankt. Den OMAHA Study Nurses S. Asendorf, N. Schödel, J. SixMerker, B. Sonnenberg und J. Wiskott gilt der Dank für verlässliche und qualitativ hervorragende Arbeit. Den Studentinnen und Studenten, die im Rahmen des Projektes gearbeitet und/oder ihre Qualifizierungsarbeiten angefertigt haben: R. Yesil, R. Diezel, S. Kühne, N. Geisemeyer, C. Stracke und schließlich den Teilnehmerinnen und Teilnehmern, ohne deren Offenheit und
Teilnahmebereitschaft diese Studie nicht möglich gewesen wäre, sagen wir ein ebenso herzliches Dankeschön. - Das Projekt MIGRANT-DEM bedankt sich bei seinen Studienteilnehmerinnen und Studienteilnehmern. - Das Projekt NEIGHBOURHOOD dankt in erster Linie den alten Menschen in den drei Untersuchungsregionen, die im Rahmen der Interviews Auskunft gegeben haben. Zudem gilt der Dank des Projektes den Mitarbeiterinnen und Mitarbeitern von Verwaltungen und Trägern sowie den Multiplikatorinnen und Multiplikatoren in den Wohngebieten, die das Vorhaben unterstützt haben. - Das Projekt INSOMNIA bedankt sich bei den Kooperationspartnern, die durch ihre Mitwirkung die Gewinnung der umfassenden Datensammlung ermöglicht haben. Ausdrücklich sollen genannt werden: Die Steuerungsgruppe und die Mitgliedseinrichtungen des Berliner Projekts, AOK BerlinBrandenburg, Arbuma Consulting/Berlin und die Interviewpartner aus den Reihen der pflegerischen Mitarbeiterinnen und Mitarbeiter, Ärztinnen und Ärzte sowie Angehörigen von Bewohnerinnen und Bewohnern der untersuchten Pflegeheime. - Der Dank des Projektes PAIN gilt dem Pflegepersonal und insbesondere den Bewohnerinnen und Bewohnern der 40 beteiligten Pflegeheime in Berlin/Brandenburg.

Interessenkonflikt. Die AMA-Projekte erklären, dass kein Interessenkonflikt vorliegt.

\section{Literatur}

1. Deutsches Netzwerk für Qualitätsentwicklung in der Pflege (DNQP) (2005) Expertenstandard Schmerzmanagement in der Pflege bei akuten und tumorbedingt chronischen Schmerzen. Entwicklung - Konsolidierung - Implementierung. Schriftenreihe des Deutschen Netzwerks für Qualitätsentwicklung in der Pflege, Fachhochschule Osnabrück

2. AGS (2002) The management of persistent pain in older persons. J Am Geriatr Soc 6(Suppl):205-224

3. Ashford S, Edmunds J, French DP (2010) What is the best way to change self-efficacy to promote lifestyle and recreational physical activity? A systematic review with meta-analysis. Br J Health Psychol 15:265-288

4. Bago d'Uva T, O'Donnell O, Doorslaer E van (2008) Differential health reporting by education level and its impact on the measurement of health inequalities among older Europeans. Int J Epidemiol 37:1375-1383

5. Baltes MM, Carstensen LL (1996) Gutes Leben im Alter: Überlegungen zu einem prozeßorientierten Metamodell erfolgreichen Alterns. Psychol Rundsch 47:199-215

6. Bandura A (1997) Self-efficacy: the exercise of control. W H Freeman, New York

7. Basler HD, Bloem R, Casser HR et al (2001) Ein strukturiertes Schmerzinterview für geriatrische Patienten. Schmerz 15:164-171

8. Beinhoff U, Hilbert V, Bittner D et al (2006) Screening for cognitive impairment: a triage for outpatient care. Dement Geriatr Cogn Disord 20:278-285

9. Beinhoff U, Riepe MW (in Vorbereitung) Demenz und Multimorbidität bei Patienten mit Migrationshintergrund. In: Kuhlmey A, Tesch-Römer C (Hrsg) Autonomie trotz Multimorbidität im Alter. Ressourcen zum Erhalt von Selbständigkeit und Selbstbestimmung. Hogrefe, Göttingen 
10. Bernard $P, C$ haraffeddine R, Frohlich KL et al (2007) Health inequalities and place: a theoretical conception of neighbourhood. Soc Sci Med 65:18391852

11. Bourdieu P (1976) Entwurf einer Theorie der Praxis. Suhrkamp, Frankfurt am Main

12. Bourdieu P (1983) Ökonomisches Kapital - Kulturelles Kapital - Soziales Kapital. In: Kreckel R (Hrsg) Soziale Ungleichheiten. Schwartz, Göttingen, S 183-198

13. Brandtstädter J, Meiniger C, Gräser H (2003) Handlungs- und Sinnressourcen: Entwicklungsmuster und protektive Effekte. Z Entwicklungspsychol Padagog Psychol 35:49-58

14. Brooks R, Rabin R, Charro F de (2003) The measurement and valuation of health status using EQ-5D: A European perspective. Kluwer Academic Publishers, Amsterdam

15. Burrows AB, Morris JN, Simon SE et al (2000) Development of a minimum Data Set-Based depression rating scale for use in nursing homes. Age Ageing 29:165-172

16. Buschke H, Kuslansky G, Katz M et al (1999) Screening for dementia with the memory impairment screen. Neurology 52:231

17. Charlson ME, Szatrowski TP, Peterson J, Gold J (1994) Validation of a combined comorbidity index. J Clin Epidemiol 47:1245-1251

18. Cimarolli VR, Reinhardt JP, Horowitz A (2006) Perceived overprotection: support gone bad? J Gerontol B Psychol Sci Soc Sci 61B:18-23

19. Delis DC, Kramer JH, Kaplan E, Ober BA (2000) California verbal learning test manual: second edition, adult version. Psychological Corporation, San Antonio

20. Dräger D, Ellert S, Kölzsch M et al (in Vorbereitung) Autonomie trotz Schmerz: Ressourcen und Barrieren in der Lebenswelt von Pflegeheimheimbewohner. In: Kuhlmey A, Tesch-Römer C (Hrsg) Autonomie trotz Multimorbidität im Alter. Ressourcen zum Erhalt von Selbständigkeit und Selbstbestimmung. Hogrefe, Göttingen

21. Evans WJ (1999) Exercise training guidelines for the elderly. Med Sci Sports Exer 31:12-17

22. Falk K, Heusinger J, Kammerer K et al (2011) Arm, alt, pflegebedürftig. Selbstbestimmungs- und Teilhabechancen im benachteiligten Quartier. edition sigma, Berlin

23. Ferrell BR (1996) Patients education and nondrug interventions. In: Ferrell BR, Ferrell BA (Hrsg) Pain in the Elderly. IASP Press, Seattle, S 35-44

24. Fiatarone MA, O'Neill EF, Ryan ND et al (1994) Exercise training and nutritional supplementation for physical frailty in very elderly people. N Engl J Med 330:1769-1775

25. Fischer T (2007) Instrumente für die Schmerzeinschätzung bei Personen mit schwerer Demenz: Hilfsmittel für die Beobachtung, aber kein Ersatz für die Fachlichkeit. Pflegezeitschrift 59:308-311

26. Flick U, Garms-Homolová V, Herrmann WJ et al (in Vorbereitung) „I can't prescribe something only because someone ask for it ..." - using mixed methods in the framework of triangulation

27. Flick U, Garms-Homolová V, Rönsch G (2010) "When they sleep, they sleep" - daytime activities and sleep disorders in nursing homes. J Health Psychol 15:755-764

28. Folstein MF, Folstein SE, McHugh PR (1975),Minimental state': a practical method for grading the cognitive state of patients for the clinician. J Psychiatr Res 12:189-198

29. Ford AB, Haug MR, Stange KC et al (2000) Sustained personal autonomy: a measure of successful aging. J Aging Health 12:470-489
30. Fortin M, Bravo G, Hudon C et al (2006) Relationship between multimorbidity and health-related quality of life of patients in primary care. Qual Life Res 15:83-91

31. Fortin M, Lapointe L, Hudon C et al (2004) Multimorbidity and quality of life in primary care: a systematic review. Health Qual Life Outcomes 2:51

32. Frederiksen K, Tariot P, De Jonghe E (1996) Minimum Data Set Plus (MDS+) scores compared with scores from five rating scales. J Am Geriatr Soc 44:305-309

33. Fried LP, Tangen CM, Walston J et al (2001) Frailty in older adults: evidence for a phenotype. J Gerontol Med Sci 56A:146-156

34. Fries BE, Simon SE, Morris JN et al (2001) Pain in US nursing homes: validating a pain scale for the minimum data set. Gerontologist 41:173-179

35. Garms-Homolová V, Flick U (in Vorbereitung) Schlafqualität und Management von Schlafstörungen im Alter. Hogrefe, Göttingen

36. Garms-Homolova V, Flick U, Kuck J et al (im Druck) Verfügbarkeit von Ressourcen zur Bewältigung von Schlafstörungen hochbetagter Pflegeheimbewohner. In: Kuhlmey A, Tesch-Römer C (Hrsg) Autonomie trotz Multimorbidität im Alter - Geht das? Eine Analyse von Ressourcen zum Erhalt der Selbständigkeit und Selbstbestimmung. Hogrefe, Göttingen

37. Garms-Homolová V, Flick U, Rönsch G (2010) Sleep disorders and activities in long term care facilities - A vicious cycle? J Health Psychol 15:744-754

38. Garms-Homolova V, Gilgen R (2000) RAI 2.0. Resident Assessment Instrument. Beurteilung, Dokumentation und Pflegeplanung in der Langzeitpflege und geriatrischen Rehabilitation. Hans Huber, Bern

39. Garms-Homolová V, Theiss K (in Vorbereitung) Aktivierung und Mobilisierung von Menschen mit Schlafstörungen. In: Garms-Homolová V, Flick U (Hrsg) Schlafqualität und Management von Schlafstörungen im Alter. Hogrefe, Göttingen

40. Garms-Homolová V, Theiss K (in Vorbereitung) Schlafstörungen als Risiko für Gesundheit und Wohlbefinden. In: Garms-Homolová V, Flick U (Hrsg) Schlafqualität und Management von Schlafstörungen im Alter. Hogrefe, Göttingen

41. Gerritsen DL, Steverink N, Frijters DH et al (2008) A revised index for social engagement for long-term care. J Gerontol Nurs 34:40-48

42. Gilmore GD, Campbell MD (1996) Needs assessment strategies for health education and health promotion. Brown \& Benchmark, Madison

43. Glaser B, Strauss A (1967) The discovery of grounded theory. Aldine, Chicago

44. Groll DL, To T, Bombardier C, Wright JG (2005) The development of a comorbidity index with physical function as the outcome. J Clin Epidemiol 58:595602

45. Herrmann WJ, Flick U (2011) Guter Schlaf aus der Sicht von Pflegeheimbewohnerinnen und Pflegeheimbewohnern. Z Allgemeinmed 87:90-94

46. Hertz J (1991) The perceived enactment of autonomy scale: measuring the potential for self-care action in the elderly. 52 (1953B), University Microfilms No. 91-28:248, Dissertation Abstracts International

47. Hertz JE (1996) Conceptualization of perceived enactment of autonomy in the elderly. Issues Ment Health Nurs 17:261-273
48. Heusinger J, Falk K, Kammerer K et al (in Vorbereitung) Chancen und Barrieren für Autonomie trotz Pflegebedarf in sozial benachteiligten Quartieren und Nachbarschaften - Projekt NEIGHBOURHOOD. In: Kuhlmey A, Tesch-Römer C (Hrsg) Autonomie trotz Multimorbidität im Alter. Ressourcen zum Erhalt von Selbständigkeit und Selbstbestimmung. Hogrefe, Göttingen

49. Holzhausen M, Fuchs J, Busch M et al (2011) Operationalizing multimorbidity and autonomy for health services research in aging populations - the OMAHA study. BMC Health Serv Res 11:47

50. Holzhausen M, Gaertner B, Martus P et al (in Vorbereitung) Operationalisierung von Multimorbidität und Autonomie für die Versorgungsforschung in alternden Populationen: Krankheitsmuster, Studienteilnahme und Lebensqualität. In: Kuhlmey A, Tesch-Römer C (Hrsg) Autonomie trotz Multimorbidität im Alter. Ressourcen zum Erhalt von Selbständigkeit und Selbstbestimmung. Hogrefe, Göttingen

51. Holzhausen M, Kuhlmey A, Martus P (2010) Individualized measurement of quality of life in older adults: Development and pilot testing of a new tool. Eur J Ageing 7:201-211

52. Holzhausen M, Scheidt-Nave C, Martus P (submitted) Validation of a new patient-generated questionnaire for quality of life in an urban sample of older women and men. Qual Life Res

53. Hutt E, Pepper GA, Vojir C et al (2006) Assessing the appropriateness of pain medication prescribing practices in nursing homes. J Am Geriatr Soc 54:231-239

54. Katz S, Down TD, Cash HR, Grotz RC (1970) Progress in the development of the index of ADL. Gerontologist 10:20-30

55. Kölzsch M, Kopke K, Fischer T et al (2011) Prescribing of inappropriate medication in nursing home residents in Germany according to a French consensus list: a cross-sectional cohort study. Pharmacoepidemiol Drug Saf 20:12-19

56. Kues H (2008) Dr. Hermann Kues: „Deutschland wird ein Land des langen Lebens - das bringt Chancen für uns alle". Pressemitteilungen des Bundesministeriums für Familie, Senioren, Frauen und Jugend (Hrsg) vom 02.09.2008, [Online] Verfügbar unter: http://www.bmfsfj.de/BMFSFJ/ Service/Archiv/16-legislatur, did=112728.html [04.10.2011]

57. Lawton MP, Brody EM (1969) Assessment of older people: Self-maintaining and instrumental activities of daily living. Gerontologist 9:179-186

58. Leonhardt C, Laekmann M (im Druck) Schmerz und Bewegungsangst im Alter. Schmerz

59. Leveille SG, Jones RN, Kiely DK et al (2009) Chronic musculoskeletal pain and the occurrence of falls in an older population. JAMA 20:2214-2221

60. Mahony F, DW B (1965) Functional Evaluation: the Barthel Index. Md State Med J 14:61-65

61. Migala S (in Vorbereitung) Mikroumwelt Heim und ihr Einfluss auf den Schlaf der Bewohnerinnen und Bewohner. In: Garms-Homolová V, Flick U (Hrsg) Schlafqualität und Management von Schlafstörungen im Alter. Hogrefe, Göttingen

62. Migala S, Röse K, Genzmer S et al (2010) Schlafstörungen Teil 2: Gut geruht? Schlafstörungen vorbeugen. Heilberufe Pflegekolleg 5

63. Migala S, Röse K, Genzmer S et al (2010) Schlafstörungen Teil 3: Möglichkeiten nicht-medikamentöser Interventionen in der pflegerischen und therapeutischen Versorgung. Heilberufe Pflegekolleg 6

64. Mor V, Branco K, Fleishman J et al (1995) The structure of social engagement among nursing home residents. J Gerontol B Psychol Sci Soc Sci 50:1-5 
65. Morris J, Murphy K, Nonemaker S et al (1995) Long-term care resident assessment instrument. Users Manual 2.0. Research Triangle Institute, Chapel Hill

66. Morris JN, Fries BE, Mehr DR et al (1994) MDS. Cognitive performance scale. J Gerontol 49:174-182

67. Morris JN, Fries BE, Morris SA (1999) Scaling ADLs within the MDS. J Gerontol A Biol Sci Med Sci 54:546-553

68. Noguchi K, Albarracín D, Durantini MR, Glasman LR (2007) Who participates in which health promotion programs? A meta-analysis of motivations underlying enrollment and retention in HIV-prevention interventions. Psychol Bull 133:955-975

69. Podsiadlo D, Richardson S (1991) The timed „Up \& $\mathrm{Go}^{\prime \prime}$ : a test of basic functional mobility for frail elderly persons. J Am Geriatr Soc 2:142-148

70. Proctor WR, Hirdes JP (2001) Pain and cognitive status among nursing home residents in Canada. Pain Res Manage 6:119-125

71. Razum O, Geiger I, Zeeb H, Ronellenfitsch U (2004) Gesundheitsversorgung von Migranten. Dtsch Arztebl 101:2882-2887

72. Rejeski WJ, Mihalko SL (2001) Physical activity and quality of life in older adults. J Gerontol A Biol Sci Med Sci 56:23-35

73. Richter S, Flick U, Kuhlmey A (2011) Alter(n) und Gesundheit. Chancen und Hürden einer disziplinübergreifenden Zusammenarbeit. GGG - Das Wissenschaftsforum in Gesundheit und Gesellschaft 2:7-15

74. Richter S, Kuhlmey A, Tesch-Römer C (in Vorbereitung) Altern(n) und Gesundheit als Herausforderung für Forschung in Verbundzusammenhängen. In: Kuhlmey A, Tesch-Römer C (Hrsg) Autonomie trotz Multimorbidität im Alter. Ressourcen zum Erhalt von Selbständigkeit und Selbstbestimmung. Hogrefe, Göttingen

75. Rönsch G, Flick U (in Vorbereitung) Schlafstörungen von Hochbetragten im Pflegeheim aus der Sicht ihrer Angehörigen. In: Garms-Homolová V, Flick U (Hrsg) Schlafqualität und Management von Schlafstörungen im Alter. Hogrefe, Göttingen

76. Ryan RM, Deci EM (2006) Self-regulation and the problem of human autonomy: Does psychology need choice, self-determination, and will? J Pers 74:1557-1585

77. Scheidt-Nave C, Richter S, Fuchs J, Kuhlmey A (2010) Herausforderungen an die Gesundheitsforschung für eine alternde Gesellschaft am Beispiel der "Multimorbidität". Bundesgesundheitsblatt 53:441-450

78. Schneekloth U (2006) Entwicklungstrends beim Hilfe- und Pflegebedarf in Privathaushalten - Ergebnisse der Infratest-Repräsentativerhebung. In: Schneekloth U, Wahl H-W (Hrsg) Selbständigkeit und Hilfebedarf bei älteren Menschen in Privathaushalten. Kohlhammer, Stuttgart, S 57-102

79. Schneider M, Stokols D (2009) Multilevel theories of behavior change: A social ecological framework. In: Shumaker SA, Ockene JK, Riekert KA (Hrsg) The handbook of health behavior change, 3. Aufl. Springer Publishing Co, New York, S 85-105

80. Schüz B, Marx C, Wurm S et al (2011) Medication beliefs predict medication adherence in older adults with multiple illness. J Psychosom Res 70:179-187

81. Schüz B, Warner LM, Wurm S et al (in Vorbereitung) Personale Ressourcen für Autonomie trotz Multimorbidität (PREFER). In: Kuhlmey A, Tesch-Römer C (Hrsg) Autonomie trotz Multimorbidität im Alter Ressourcen zum Erhalt von Selbständigkeit und Selbstbestimmung. Hogrefe, Göttingen
82. Schüz B, Wurm S, Ziegelmann JP et al (2011) Changes in functional health, changes in medication beliefs and medication adherence. Health Psychol 30:31-39

83. Schwarzer R (2008) Wahrgenommene Autonomie im Alter. http://userpage.fu-berlin.de/ health/autonomie.htm. Zugegriffen: 4. Okt. 2011

84. Sibley A, Kersten P, Ward CD et al (2006) Measuring autonomy in disabled people: validation of a new scale in a UK population. Clin Rehab 30:793-803

85. Smith DE (2005) Institutional ethnography: a sociology for the people. Altamira, Lanham

86. Statistik Berlin Brandenburg (2011) Einbürgerungen, Ausländer, Basisdaten 2010. Amt für Statistik Berlin Brandenburg. http://www.statistik-berlin-brandenburg.de/BasisZeitreiheGrafik/Bas-Einbuergerungen_Auslaender.asp? Ptyp $=300 \&$ Sageb=12005\&creg=BBB\&anzwer=5. Zugegriffen: 4 . Okt. 2011

87. Statistisches Bundesamt [Federal Statistical Office] (2011) Pflegestatistik 2009: Pflege im Rahmen der Pflegeversicherung, Deutschlandergebnisse. Statistisches Bundesamt, Wiesbaden

88. Statistisches Bundesamt [Federal Statistical Office] (2010) Statistisches Jahrbuch 2010 für die Bundesrepublik Deutschland [Statistical Yearbook 2010 for the Federal Republic of Germany]. Statistisches Bundesamt [Federal Statistical Office], Wiesbaden, Germany

89. Taylor SE (2007) Social support. In: Friedman HS Silver RC (Hrsg) Foundations of health psychology. Oxford University Press, New York, S 145-171

90. Akker M van den, Buntinx F, Knottnerus JA (1996) Comorbidity or multimorbidity: what's in a name? A review of literature. Eur J Gen Pract 2:65-70

91. Akker $M$ van den, Buntinx F, Metsemakers JFM et al (1998) Multimorbidity in general practice: prevalence, incidence, and determinants of co-occurring chronic and recurrent diseases. J Clin Epidemiol 51:367-375

92. Warner LM, Ziegelmann JP, Schüz B et al (2011) Maintaining autonomy despite multimorbidity: self-efficacy and the two faces of social support. Eur J Ageing 8:3-12

93. Warner LM, Ziegelmann JP, Schüz B et al (im Druck) Synergistic effect of social support and self-efficacy on physical exercise in older adults. J Aging Phys Act 19:249-61

94. Whitbourne SK (2001) Differential aging. In: International encyclopedia of social and behavioral sciences. Pergamon, Oxford, S 3654-3658

95. Wurm S, Schöllgen I, Tesch-Römer C (2010) Gesundheit. In: Motel-Klingebiel A, Wurm S, TeschRömer C (Hrsg) Altern im Wandel: Befunde des Deutschen Alterssurveys (DEAS). Kohlhammer, Stuttgart, S 114-117 


\section{Hier steht eine Anzeige.}

Springer 